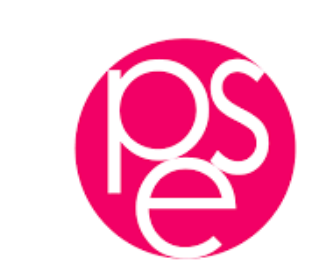

PARISSCHOOL OF ECONOMICS
ECOLE D'ECONOMIE DE PARIS

WORKING PAPER N ${ }^{\circ} 2017-26$

Hopf Bifurcation from New-Keynesian Taylor Rule to Ramsey Optimal Policy

\author{
Jean-Bernard Chatelain \\ Kirsten Ralf
}

JEL Codes: C61, C62, E43, E44, E47, E52, E58

Keywords: Bifurcations, Taylor Rule, Taylor Principle, New-Keynesian Model, Ramsey Optimal Policy

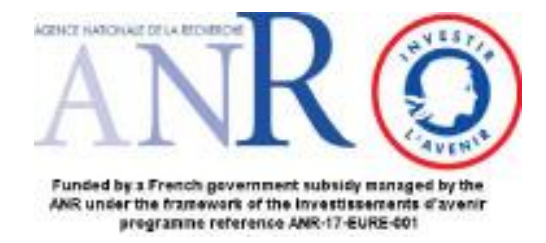




\title{
HOPF BIFURCATION FROM NEW-KEYNESIAN TAYLOR RULE TO RAMSEY OPTIMAL POLICY
}

\author{
Jean-Bernard Chatelain*and Kirsten Ralf ${ }^{\dagger}$ \\ Post-Print: Macroeconomic Dynamics, 17th January 2020, Pages 1-33. \\ https://doi.org/10.1017/S1365100519001032
}

February 15, 2020

\begin{abstract}
This paper compares different implementations of monetary policy in a newKeynesian setting. We can show that a shift from Ramsey optimal policy under short-term commitment (based on a negative feedback mechanism) to a Taylor rule (based on a positive feedback mechanism) corresponds to a Hopf bifurcation with opposite policy advice and a change of the dynamic properties. This bifurcation occurs because of the ad hoc assumption that interest rate is a forward-looking variable when policy targets (inflation and output gap) are forward-looking variables in the new-Keynesian theory.

JEL classification numbers: C61, C62, E43, E44, E47, E52, E58.

Keywords: Bifurcations, Taylor Rule, Taylor Principle, New-Keynesian Model, Ramsey Optimal Policy.
\end{abstract}

*Paris School of Economics, Université Paris I Pantheon Sorbonne, 48 Boulevard Jourdan 75014 Paris. Email: jean-bernard.chatelain@univ-paris1.fr

${ }^{\dagger}$ ESCE International Business School, Inseec U Research Center, 10 rue Sextius Michel, 75015 Paris, Email: Kirsten.Ralf@esce.fr. 


\section{INTRODUCTION}

The question of how to conduct monetary policy is one of the most discussed questions in macroeconomic theory. In this paper, we will compare Ramsey optimal policy which is based on a negative feedback mechanism to a Taylor rule which is based on a positive feedback mechanism. Both settings are analyzed in the same New-Keynesian benchmark model with households' intertemporal optimization of consumption and a New-Keynesian Phillips curve.

On the one hand, negative feedback is the core mechanism for stabilizing dynamic systems with optimal control (Aström and Kumar (2014), Chatelain and Ralf (2019b)). On the other hand, determinacy, as required by rational expectations theory, is often associated with a positive feedback mechanism in the New-Keynesian setting. Barnett and Chen (2015) and Barnett and Duzhak (2008, 2010), therefore, emphasize the importance of bifurcations in the reference New-Keynesian macroeconomic model (Gali (2015)). The Taylor rule parameters (the response of the interest rate to inflation or to the output gap) are bifurcation parameters. A small change of their values may lead to big changes of the dynamic path, from stability to instability and conversely.

The aim of this paper is to clarify these issues further, extending the bifurcation results of Barnett and Duzhak (2008 and 2010) on the closed economy New-Keynesian model. We use Wonham's (1967) pole placement theorem to locate linear feedback rule parameters for two basic types of policy: New-Keynesian Taylor rule and Ramsey optimal policy.

For Ramsey optimal policy under quasi-commitment, the policy targets (inflation and output gap) are forward-looking. Optimal behavior implies that the policy instruments (the interest rate and its lag) are optimally predetermined, using initial transversality conditions. This leads to negative feedback Taylor rule parameters since higher inflation would lead the Fed to set interest rates in a way that produces lower future inflation. This mechanism is in the spirit of Keynesian stabilization theory.

The private sector agents have a unique optimal anchor of forward-looking output gap and inflation on the unique optimal initial values of the observable central bank funds rate and its lag. With Ramsey optimal policy, negative feedback Taylor rule parameters are compatible with determinacy, which corresponds to the uniqueness of the path of forward-looking variables.

In the New-Keynesian model, the only equation which is not derived from optimal intertemporal rational choice is the Taylor rule. Hence, it is the only equation where the researcher writing down the model can arbitrarily decide if the variable whose behavior is governed by the Taylor rule equation, the central bank's funds rate, is predetermined or forward-looking. This ad hoc theoretical assumption is an input in order to apply Blanchard and Kahn's (1980) determinacy condition. If the central bank's funds rate is assumed to be forward-looking for forward-looking inflation and output gap, positive feedback Taylor rule parameters lead to determinacy, with only one possible value of inflation suiTable for the central bank. As Cochrane (2011) states: "In New-Keynesian models, higher inflation leads the Fed to set interest rates in a way that produces even higher future inflation. For only one value of inflation today will inflation fail to explode."

Inflation and output gap are forward-looking variables that need to be anchored on predetermined variables. If the central bank's funds rate is a forward-looking variable, it cannot be an anchor. Hence, the private sector agents are assumed to anchor inflation and output gap on non-observable variables, which are two ad hoc autoregressive 
predetermined shocks: a cost-push shock and a productivity shock.

We can show that the shift from Ramsey optimal policy, with two stable eigenvalues, to a Taylor rule, with two unstable complex conjugate eigenvalues, corresponds to a Hopf bifurcation which is only driven by the choice of a theoretical assumption by researchers on central bank's funds rate as being optimally predetermined variables (Ramsey optimal policy) or ad hoc forward-looking variable (Taylor rule).

The ad hoc assumption that policy instrument should be forward-looking instead of backward-looking when the policy targets are forward-looking is the origin of the bifurcation of the dynamic systems when assuming New-Keynesian Taylor rule in place of Ramsey optimal policy.

Hence, our policy recommendation would be to use Ramsey optimal policy with a reduced form negative feedback Taylor rule. For the monetary transmission channel, empirical work may confirm the relevance of a delayed cost-of-capital channel of monetary policy where interest rate is negatively correlated with future output as opposed to an intertemporal substitution channel where the interest rate is positively correlated with future output gap. If this is the case, a negative feedback Taylor rule parameter of the response of interest rate to output gap is positive. Else, it would be negative.

The rest of the paper is organized as follows: In Section 2, the model and its dynamic properties are presented, including Barnett and Duzhak's $(2008,2010)$ bifurcations frontier. In Section 3, Ramsey optimal policy and a Taylor rule are compared. Section 4 shows that a shift from Ramsey optimal policy to a New-Keynesian Taylor rule corresponds to a Hopf bifurcation. In Section 5, we analyze the consequences when changing the assumptions on the exogenous shocks. Section 6 concludes.

\section{BIFURCATIONS IN THE NEW-KEYNESIAN MODEL}

\subsection{The New-Keynesian Model}

The New-Keynesian private sector's four-equations model is written with all variables as log-deviations of an equilibrium (Gali (2015)). In the representative household's intertemporal substitution consumption Euler equation, the expected future output gap $E_{t} x_{t+1}$ is positively correlated with the real rate of interest, equal to the nominal rate $i_{t}$ minus expected inflation $E_{t} \pi_{t+1}$. The intertemporal elasticity of substitution (IES) $\gamma=1 / \sigma$ is a measure of the responsiveness of the growth rate of consumption to the interest rate, usually considered to be smaller than one. It is the inverse of $\sigma$, the relative fluctuation aversion or the relative degree of resistance to intertemporal substitution of consumption, which measures the strength of the preference for smoothing consumption over time, usually considered to be larger than one.

$$
x_{t}=E_{t} x_{t+1}-\gamma\left(i_{t}-E_{t} \pi_{t+1}\right)+z_{t} \text { with } \gamma>0 .
$$

A non-controllable exogenous stationary and predetermined variable $z_{t}$ is autoregressive of order one $\left(0<\left|\rho_{z, x}\right|<1\right)$ where $\varepsilon_{z, t}$ are zero-mean, normally, independently and identically distributed additive disturbances. The initial value of the predetermined forcing variable is given.

$$
z_{t}=\rho_{z} z_{t-1}+\varepsilon_{z, t}, z_{0} \text { given. }
$$

In the New-Keynesian Phillips curve, expected inflation $E_{t} \pi_{t+1}$ is negatively correlated 
with the current output gap $x_{t}$ with a sensitivity $-\kappa<0$.

$$
\pi_{t}=\beta E_{t} \pi_{t+1}+\kappa x_{t}+u_{t} \text { with } 1>\beta>0 \text { and } \kappa>0 .
$$

Sign restrictions are such that parameters $\gamma, \beta, \kappa$ are all strictly positive. A noncontrollable exogenous stationary and predetermined variable cost-push shock $u_{t}$ is autoregressive of order one $\left(0<\left|\rho_{u}\right|<1\right)$ where $\varepsilon_{u, t}$ are zero-mean, normally, independently and identically distributed additive disturbances. The initial value of the predetermined cost-push shock $u_{0}$ is given.

$$
u_{t}=\rho_{u} u_{t-1}+\varepsilon_{u, t}, u_{0} \text { given. }
$$

Firstly, the controllable dynamics of the New-Keynesian model with its feedback Taylor rule can be written as follows:

$$
\left(\begin{array}{c}
E_{t} x_{t+1} \\
E_{t} \pi_{t+1}
\end{array}\right)=\underbrace{\left(\begin{array}{cc}
1+\frac{\gamma \kappa}{\beta} & -\frac{\gamma}{\beta} \\
-\frac{\kappa}{\beta} & \frac{1}{\beta}
\end{array}\right)}_{=\mathbf{A}_{y y}} \underbrace{\left(\begin{array}{c}
x_{t} \\
\pi_{t}
\end{array}\right)}_{=\mathbf{y}_{t}}+\underbrace{\left(\begin{array}{l}
\gamma \\
0
\end{array}\right)}_{=\mathbf{B}_{y}} i_{t}+\underbrace{\left(\begin{array}{cc}
-1 & \frac{\gamma}{\beta} \\
0 & -\frac{1}{\beta}
\end{array}\right)}_{=\mathbf{A}_{y z}} \underbrace{\left(\begin{array}{l}
z_{t} \\
u_{t}
\end{array}\right)}_{=\mathbf{z}_{t}} .
$$

The matrix notation in bold below corresponds to the notation of the augmented linear quadratic regulator (LQR) in Anderson, Hansen, McGrattan and Sargent (1996, p.203) and Hansen and Sargent (2007). The two policy targets, the output gap $x_{t}$ and inflation $\pi_{t}$, are two-time-step Kalman controllable by a single policy instrument, the interest rate $i_{t}$ and its first lag $i_{t-1}$, if both the intertemporal elasticity of substitution and the slope of the New-Keynesian Phillips curve are different from zero: $\gamma \neq 0$ which implies $\frac{\partial E_{t}\left(x_{t+1}\right)}{\partial i_{t}} \neq 0$ and $\kappa=\frac{\partial E_{t+1}\left(\pi_{t+2}\right)}{\partial x_{t+1}} \neq 0$. This is found computing the rank of Kalman controllability matrix:

$$
\begin{aligned}
\operatorname{rank}\left(\mathbf{B}_{y}, \mathbf{A}_{y y} \mathbf{B}_{y}\right) & =\operatorname{rank}\left(\left(\begin{array}{l}
\gamma \\
0
\end{array}\right),\left(\begin{array}{cc}
1+\frac{\gamma \kappa}{\beta} & -\frac{\gamma}{\beta} \\
-\frac{\kappa}{\beta} & \frac{1}{\beta}
\end{array}\right)\left(\begin{array}{l}
\gamma \\
0
\end{array}\right)\right) \\
& =\operatorname{rank}\left(\begin{array}{cc}
\gamma & \gamma\left(\frac{\kappa}{\beta} \gamma+1\right) \\
0 & -\frac{\kappa}{\beta} \gamma
\end{array}\right)=2 \text { if } \gamma \neq 0 \text { and } \kappa \neq 0 .
\end{aligned}
$$

If the intertemporal elasticity of substitution is zero $\gamma=0$, the rank of the Kalman controllability matrix is zero. Then, the output gap is not controllable by the interest rate in the first period. Even if the one-period ahead output gap can control two-period ahead future inflation $(\kappa \neq 0)$, as the current policy instrument (the interest rate) cannot control the one-period ahead output gap, the interest rate cannot control the two-period ahead inflation.

If the intertemporal elasticity of substitution is different from zero $\gamma \neq 0$ and $\kappa=0$, the rank of Kalman controllability matrix is one. The output gap is controllable by the interest rate in the first period. But the output gap next period cannot control two periods ahead inflation $(\kappa=0)$, so that the current period interest rate cannot control two periods ahead inflation.

If the intertemporal elasticity of substitution is different from zero $\gamma \neq 0$ and the slope of the New-Keynesian Phillips curve is different from zero $\kappa \neq 0$, the rank of Kalman controllability matrix is two. The first period output gap is controllable by the interest 
rate. The first-period ahead output gap controls two-period ahead inflation. Hence, there is a non-zero correlation between expected two-period ahead inflation and current interest, $\frac{\partial E_{t+1}\left(\pi_{t+2}\right)}{\partial i_{t}}=\frac{\partial E_{t+1}\left(\pi_{t+2}\right)}{\partial E_{t}\left(x_{t+1}\right)} \frac{\partial E_{t}\left(x_{t+1}\right)}{\partial i_{t}} \neq 0$. The fact that the interest rate does not show up in the New-Keynesian Phillips curve implies that the effect of the interest rate has to go through a first-period effect on the output gap, so that a change on interest rate only shows up in two-period ahead inflation. The positive correlation of the interest rate on the one-time step expected output gap $(\gamma>0)$ on the first period is followed on the second-period negative correlation of the one-period ahead output gap on two-period ahead expected inflation $(-\kappa>0)$.

$$
\frac{\partial E_{t+1}\left(\pi_{t+2}\right)}{\partial i_{t}}=\frac{\partial E_{t+1}\left(\pi_{t+2}\right)}{\partial E_{t}\left(x_{t+1}\right)} \frac{\partial E_{t}\left(x_{t+1}\right)}{\underset{<0}{\partial i_{t}}}<0 .
$$

Kalman (1960) controllability is the generalization of Tinbergen's (1952) principle for a static linear system of equations ( $n$ policy instruments can control $n$ policy targets in a single period) to linear dynamic systems. One policy instrument and its first lag can control two policy targets in two periods.

Secondly, the dynamics of the non-controllable exogenous forcing variables can be stated as:

$$
\left(\begin{array}{c}
z_{t+1} \\
u_{t+1}
\end{array}\right)=\underbrace{\left(\begin{array}{cc}
\rho_{z} & 0 \\
0 & \rho_{u}
\end{array}\right)}_{=\mathbf{A}_{z z}}\left(\begin{array}{c}
z_{t} \\
u_{t}
\end{array}\right)+\left(\begin{array}{c}
\varepsilon_{z, t} \\
\varepsilon_{u, t}
\end{array}\right) .
$$

Thirdly, the feedback linear policy rule in the augmented linear quadratic regulator responds also to forcing variables (Anderson, Hansen, McGrattan and Sargent (1996, p.203), Hansen and Sargent (2007)):

$$
i_{t}=\underbrace{\left(\begin{array}{ll}
F_{x} & F_{\pi}
\end{array}\right)}_{=\mathbf{F}_{y}}\left(\begin{array}{c}
x_{t} \\
\pi_{t}
\end{array}\right)+\underbrace{\left(\begin{array}{ll}
F_{z} & F_{u}
\end{array}\right)}_{=\mathbf{F}_{z}}\left(\begin{array}{c}
z_{t} \\
u_{t}
\end{array}\right) .
$$

\subsection{Dynamic Properties}

The dynamic properties of the New-Keynesian model depend on the eigenvalues of the system linearized around the steady state. In this section we show that a unique relationship between the two Taylor rule parameters $\left(F_{\pi}, F_{x}\right)$ and the trace and the determinant of the closed-loop matrix exist. This will allow us to analyze determinacy and the existence of bifurcations by looking at the Taylor rule parameters that have an economic interpretation which is sometimes missing for the trace and determinant of a dynamic system. Additionally to Barnett and Duzhak's (2008) Hopf bifurcation border and Barnett and Duzhak's (2010) flip bifurcation border, the last missing side of the stability triangle which corresponds to the saddle-node bifurcation border can be identified. This specific relation in the New-Keynesian model is a particular case of the mapping of feedback rule parameters with closed-loop eigenvalues of controllable linear system demonstrated by Wohnam (1967) pole placement theorem. We use Azariadis' (1993, pp. 63-67) conditions on the trace and determinant for a discrete dynamic system of dimension 2 in order to find the bifurcation limits of the stability triangle in the plane of Taylor rule parameters.

The closed-loop matrix $\mathbf{A}_{y y}+\mathbf{B}_{y} \mathbf{F}_{y}$ (denoted $\mathbf{A}+\mathbf{B F}$ in what follows) of the controllable part of the New-Keynesian model is: 


$$
\left(\begin{array}{cc}
1+\frac{\gamma \kappa}{\beta} & -\frac{\gamma}{\beta} \\
-\frac{\kappa}{\beta} & \frac{1}{\beta}
\end{array}\right)+\left(\begin{array}{l}
\gamma \\
0
\end{array}\right)\left(\begin{array}{ll}
F_{x} & F_{\pi}
\end{array}\right)=\left(\begin{array}{cc}
1+\frac{\gamma \kappa}{\beta}+\gamma F_{x} & -\frac{\gamma}{\beta}+\gamma F_{\pi} \\
-\frac{\kappa}{\beta} & \frac{1}{\beta}
\end{array}\right)
$$

The characteristic polynomial of the closed-loop matrix $\mathbf{A}+\mathbf{B F}$ of the New-Keynesian model is a function of its trace $T$ and determinant $D$ leading to two eigenvalues $\lambda_{1}$ and $\lambda_{2}$ :

$$
p(\lambda)=\operatorname{det}(\mathbf{A}+\mathbf{B F}-\lambda \mathbf{I})=\lambda^{2}-T \lambda+D=\left(\lambda-\lambda_{1}\right)\left(\lambda-\lambda_{2}\right)=0 .
$$

The eigenvalues (the roots of the characteristic polynomial) are non-linear functions of the trace and the determinant. We either have two ordered real eigenvalues or two complex conjugate eigenvalues:

$$
\begin{aligned}
& \lambda_{1}=\frac{T-\sqrt{T^{2}-4 D}}{2}<\lambda_{2}=\frac{T+\sqrt{T^{2}-4 D}}{2} \text { if } \Delta=T^{2}-4 D \geq 0 \\
& \lambda_{1}=\frac{T-i \sqrt{4 D-T^{2}}}{2} \text { and } \lambda_{2}=\bar{\lambda}_{1}=\frac{T+i \sqrt{4 D-T^{2}}}{2} \text { if } \Delta=T^{2}-4 D<0 .
\end{aligned}
$$

The trace $T$ and determinant $D$ of the closed-loop matrix $\mathbf{A}+\mathbf{B F}$, however, are affine functions of the Taylor rule parameters $F_{x}$ and $F_{\pi}$, where constants are, respectively, the trace $T(\mathbf{A})$ and the determinant $D(\mathbf{A})$ of the open-loop matrix $\mathbf{A}$ :

$$
\begin{aligned}
& T(\mathbf{A}+\mathbf{B F})=1+\frac{1}{\beta}+\frac{\gamma \kappa}{\beta}+\gamma F_{x}=T(\mathbf{A})+\gamma F_{x}, \\
& D(\mathbf{A}+\mathbf{B F})=\frac{1}{\beta}+\frac{\gamma}{\beta} F_{x}+\frac{\gamma \kappa}{\beta} F_{\pi}=D(\mathbf{A})+\frac{1}{\beta} \gamma F_{x}+\frac{\kappa}{\beta} \gamma F_{\pi}, \\
& \text { with } T(\mathbf{A})=1+\frac{1}{\beta}+\frac{\gamma \kappa}{\beta} \text { and } D(\mathbf{A})=\frac{1}{\beta} .
\end{aligned}
$$

Conversely, the Taylor rule parameters $F_{x}$ and $F_{\pi}$ are linear functions of the difference of the closed-loop and the open-loop trace $T(\mathbf{A}+\mathbf{B F})-T(\mathbf{A})$ and of the difference of the closed-loop and the open-loop determinant $D(\mathbf{A}+\mathbf{B F})-D(\mathbf{A})$ and affine functions of the closed-loop trace and determinant:

$$
\begin{aligned}
F_{x} & =\frac{1}{\gamma}(T(\mathbf{A}+\mathbf{B F})-T(\mathbf{A}))=\frac{1}{\gamma} T(\mathbf{A}+\mathbf{B F})-\frac{1}{\gamma}\left(1+\frac{1}{\beta}+\frac{\gamma \kappa}{\beta}\right), \\
F_{\pi} & =-\frac{1}{\gamma \kappa}(T(\mathbf{A}+\mathbf{B F})-T(\mathbf{A}))+\frac{\beta}{\gamma \kappa}(D(\mathbf{A}+\mathbf{B F})-D(\mathbf{A})) \\
& =-\frac{1}{\gamma \kappa} T(\mathbf{A}+\mathbf{B F})+\frac{\beta}{\gamma \kappa} D(\mathbf{A}+\mathbf{B F})+\frac{1}{\beta}+\frac{1}{\gamma \kappa \beta} .
\end{aligned}
$$

In classic control, these feedback rule parameters are corresponding to a "pole placement" with desired closed-loop eigenvalues defined by the parameters of the characteristic polynomial, which are in dimension 2 , the trace $T(\mathbf{A}+\mathbf{B F})$ and the determinant $D(\mathbf{A}+\mathbf{B F})$. This relation can also be found using the canonical form of the dynamic system or using Ackermann's (1972) formula (see appendix B).

In Azariadis (1993, chapter 8), a stability triangle with Hopf $(D=1$ and $\Delta<0)$, 
flip $(p(-1)=0)$ and saddle-node $(p(1)=0)$ bifurcation borders and a quadratic function delimiting complex conjugate versus non-complex solutions (discriminant $\Delta=0$ ) are described in the plane of the trace and determinant $(T, D)$. This defines areas with zero, one or two stable eigenvalues $\left(\lambda_{1}, \lambda_{2}\right)$ in the plane $(T, D)$. We use Azariadis' (1993) insights to compute bifurcation borders in the plane of Taylor rule bifurcation parameters $\left(F_{\pi}, F_{x}\right)$. An example for given parameter values of the New-Keynesian model is depicted in Figure 1 with the numerical values for the corresponding points and the values of the rule parameters and the eigenvalues given in Table 1 . Inside the triangle ABC the eigenvalues have modulus smaller than 1 , and outside the triangle at least one eigenvalue is larger than one in absolute value. The dotted parabola through the points $\mathrm{A}, \Omega$, and $\mathrm{B}$ corresponds to a discriminant equal to zero with complex eigenvalues on the right-hand side of the parabola and real eigenvalues on its left-hand side. The center $\Omega$ has both eigenvalues equal to zero $\left(\lambda_{1}=\lambda_{2}=0\right)$ and provides the Taylor rule parameters with the fastest stabilization.

$$
\Delta=0 \Leftrightarrow \frac{1}{\beta}+\frac{\gamma}{\beta} F_{x}+\frac{\gamma \kappa}{\beta} F_{\pi}=\frac{1}{4}\left(1+\frac{1}{\beta}+\frac{\gamma \kappa}{\beta}+\gamma F_{x}\right)^{2} .
$$

On the segment connecting the points A and B both eigenvalues are conjugate complex and have modulus one. This is the case when the determinant is equal to one:

$$
D(\mathbf{A}+\mathbf{B F})=1 \Leftrightarrow F_{x}=\frac{\beta-1}{\gamma}-\kappa F_{\pi}
$$

Above this line, that is for policy parameters $F_{x}>\frac{\beta-1}{\gamma}-\kappa F_{\pi}$, the eigenvalues have modulus larger than one and below this line, that is for policy parameters $F_{x}<\frac{\beta-1}{\gamma}-\kappa F_{\pi}$, the eigenvalues have modulus smaller than one. Crossing this line from inside the triangle corresponds to a Hopf bifurcation, see also Barnett and Duzhak (2008).

To analyze the real eigenvalues, we look at the characteristic polynomial:

$$
\begin{aligned}
& p(a)=\left(a-\lambda_{1}\right)\left(a-\lambda_{2}\right)>0 \Leftrightarrow a<\lambda_{1}<\lambda_{2} \text { or } \lambda_{1}<\lambda_{2}<a, \\
& p(a)=\left(a-\lambda_{1}\right)\left(a-\lambda_{2}\right)<0 \Leftrightarrow \lambda_{1}<a<\lambda_{2} .
\end{aligned}
$$

The two lines $p(1)=0$ and $p(-1)=0$ divide the plane into four regions as shown in Figures 1 and 2. They cross at point $\mathrm{C}\left(\lambda_{1}=-1\right.$ and $\left.\lambda_{2}=1\right)$.

On the line going through the points $\mathrm{A}$ and $\mathrm{C}$, at least one of the real eigenvalues is equal to one. It is characterized by

$$
\begin{aligned}
& p(1)=1-T(\mathbf{A}+\mathbf{B F})+D(\mathbf{A}+\mathbf{B F})=0 \\
\Leftrightarrow & F_{x}=\frac{\kappa}{1-\beta}\left(1-F_{\pi}\right) \text { or } F_{\pi}=1-\left(\frac{1-\beta}{\kappa}\right) F_{x} .
\end{aligned}
$$

If $F_{x}>0$ but not too large, $F_{\pi}$ can be slightly below one. If $F_{x}<0, F_{\pi}$ is strictly larger than one: it satisfies the Taylor principle. When the discount factor $\beta$ is close to one, the slope of the line is close to infinity (vertical line), so that this condition is close to the Taylor principle condition $F_{\pi}>1$. It goes through the point $\mathrm{A}\left(\lambda_{1}=\lambda_{2}=1\right)$ of Table 1 which is the point of intersection of the parabola of a discriminant equal to zero $(\Delta=0)$ and a determinant equal to one $D=\lambda_{1} \lambda_{2}=1$. It goes through the point $\mathrm{C}$ $\left(\lambda_{1}=-1\right.$ and $\left.\lambda_{2}=1\right)$ of Table 1 , point of intersection of the line $p(-1)=0$. 
On the right-hand side of the line $p(1)=0$ and inside the triangle, both eigenvalues are smaller than one in absolute value. Crossing the line from the left leads to one eigenvalue larger than one and the other one smaller than one, a saddle-node bifurcation.

Similarly,

$$
p(-1)=1+T+D=0 \Rightarrow F_{x}=-\frac{2}{\gamma}-\frac{\kappa}{1+\beta}-\frac{\kappa}{1+\beta} F_{\pi}
$$

which goes through the points $\mathrm{C}\left(\lambda_{1}=-1\right.$ and $\left.\lambda_{2}=1\right)$ and $\mathrm{B}\left(\lambda_{1}=\lambda_{2}=-1\right)$, see Table 1. Above this line and inside the triangle, we have $p(-1)>0$, with both roots smaller than 1 in absolute value. Below this line $p(-1)<0$, one of the eigenvalues is smaller than -1 and the other eigenvalue is larger than -1 . Crossing this line from inside the triangle corresponds to a flip bifurcation, see also Barnett and Duzhak (2010).

The parameter values in Table 1 correspond to some estimated values: $\gamma=0.5$ for the intertemporal elasticity in the USA (Havranek et al. (2015)) and $\kappa=0.1$ for the slope of the New-Keynesian Phillips curve $\kappa=0.1$ (Mavroeidis et al. (2014)).

Table 1: Stability triangle with center and point of laissez-faire (origin) of the New-Keynesian model $(\gamma=0.5, \kappa=0.1, \beta=0.99)$, with $T(\mathbf{A})=1+\frac{1}{\beta}+\frac{\gamma \kappa}{\beta}=2$. $06>2$.

\begin{tabular}{|l|l|l|l|l|l|l|}
\hline & $\lambda_{1}$ & $\lambda_{2}$ & $\lambda_{1}+\lambda_{2}$ & $\lambda_{1} \lambda_{2}$ & $F_{\pi}$ & $F_{x}$ \\
\hline $\mathrm{A}$ & 1 & 1 & 2 & 1 & $\frac{-(2-T(\mathbf{A}))+\beta\left(1-\frac{1}{\beta}\right)}{\gamma \kappa}=1.01$ & $\frac{2-T(\mathbf{A})}{\gamma}=-0.12$ \\
\hline $\mathrm{B}$ & -1 & -1 & -2 & 1 & $\frac{-(-2-T(\mathbf{A}))+\beta\left(1-\frac{1}{\beta}\right)}{\gamma \kappa}=81.0$ & $\frac{-2-T(\mathbf{A})}{\gamma}=-8.12$ \\
\hline $\mathrm{C}$ & -1 & 1 & 0 & -1 & $\frac{T(\mathbf{A})+\beta\left(-1-\frac{1}{\beta}\right)}{\gamma \kappa}=1.41$ & $-\frac{T(\mathbf{A})}{\gamma}=-4.12$ \\
\hline$\Omega$ & 0 & 0 & 0 & 0 & $\frac{T(\mathbf{A})+\beta\left(-\frac{1}{\beta}\right)}{\gamma \kappa}=21$ & $-\frac{T(\mathbf{A})}{\gamma}=-4.12$ \\
\hline $\mathrm{O}$ & $0<\lambda_{1}<1$ & $1<\frac{1}{\lambda_{1} \beta}$ & $T(\mathbf{A})$ & $\frac{1}{\beta}$ & 0 & $\frac{T(\mathbf{A})-T(\mathbf{A})}{\gamma}=0$ \\
\hline
\end{tabular}

Figure 1 represents bifurcation lines delimiting the number of stable eigenvalues for the New-Keynesian model for positive sign restrictions on monetary policy transmission mechanism. The case of negative sign restrictions on monetary policy transmission mechanism is treated separately in section 2.4 (Figure 2). Figure 1 shows how the plane is divided by the four reference lines $p(-1)=0, p(1)=0, \Delta=0$ and $D=1$ into eight regions. We start in the upper left quadrant and move counter-clockwise.

Region 1: On the left of the line $\mathrm{AC}$ and above the line $\mathrm{BC}, p(1)<0$ and $p(-1)>0$. Both eigenvalues are on the same side of -1 and on different sides of 1 . The only possibility is $-1<\lambda_{1}<1<\lambda_{2}$. The steady state is a saddle-point. This area includes the origin 0 which corresponds to the laissez-faire open-loop equilibrium, where both Taylor rule parameters are equal to zero.

Region 2: On the left of the line $\mathrm{AC}$ and below the line BC, $p(1)<0$ and $p(-1)<0$. The only possibility is $\lambda_{1}<-1$ and $1<\lambda_{2}$. Both eigenvalues are on different sides of 1 and on different sides of -1 . The steady state is a source.

Region 3: On the right of the line $\mathrm{AC}$ and below the line $\mathrm{BC}, p(1)>0$ and $p(-1)<0$. Both eigenvalues are on the same side of 1 and on different sides of -1 . The only possibility is $\lambda_{1}<-1<\lambda_{2}<1$. The steady state is a saddle-point.

Region 4: On the right of the line $\mathrm{AC}$ and above the line $\mathrm{BC}, p(1)>0$ and $p(-1)>0$. This region is split into five regions as follows:

(4.1): Above the line BC and below the parabola: Both eigenvalues are real with $-1<\lambda_{1}<\lambda_{2}<1$. The steady state is a sink. 
(4.2): Above the parabola and below the line AB: Both eigenvalues are conjugate complex with modulus smaller than one $\left|\lambda_{1}\right|=\left|\lambda_{2}\right|<1$, the steady state is a sink.

(4.3): Above the line $\mathrm{AB}$ and inside the parabola: Both eigenvalues are conjugate complex with modulus larger than one $\left|\lambda_{1}\right|=\left|\lambda_{2}\right|>1$, the steady state is a source.

(4.4): Outside the parabola and on the right of the line AC: Both eigenvalues are real and larger than one with $1<\lambda_{1}<\lambda_{2}$. The steady state is a source.

(4.5): The last region corresponds to the area outside the triangle above the line BC below the parabola. Here, both eigenvalues are negative $\lambda_{1}<\lambda_{2}<-1$, with their sum (trace) below -2 .

In the stability triangle $\mathrm{ABC}$, the Taylor principle is satisfied $F_{\pi}>1$ and the output gap Taylor rule parameter is negative: $F_{x}<0$.

Crossing the lines $p(1)=0$ or $p(-1)=0$ outside the stability triangle corresponds to another type of bifurcations between unstable dynamic systems in the sense that number of stable eigenvalues shifts from one to zero or the reverse. In particular, for positive Taylor rule parameters $F_{\pi} \geq 0$ and $F_{x} \geq 0$, the laissez-faire regime with zero Taylor rule parameters (the origin of Figure 1) corresponds to a case such that $-1<\lambda_{1}<1<\lambda_{2}$, to the left of the line $p(1)=0$. This laissez-faire ("open-loop") private sector's model is described by the Fed following a fixed interest rate target or peg: $i_{t}-i^{*}=0$. An increase of the inflation Taylor rule parameter $F_{\pi}$ shifts the dynamic system from the area where $-1<\lambda_{1}<1<\lambda_{2}$ with $p(1)<0$ up to crossing the line $p(1)=0$ to a an area where $1<\lambda_{1}<\lambda_{2}$ with $p(1)>0$. This is a saddle-node bifurcation between unstable systems which are both having at least one unstable eigenvalue $\lambda_{2}$.

Proposition 1. For strictly positive intertemporal elasticity of substitution $\gamma>0$ and a strictly positive slope of the New-Keynesian Phillips curve $\kappa>0$ and a positive discount factor below one $0<\beta \leq 1$, a necessary condition for having two stable eigenvalues $\left(\left|\lambda_{1}\right|<1\right.$ and $\left.\left|\lambda_{2}\right|<1\right)$ in the closed-loop transition matrix of the New-Keynesian model is that rule parameters $\left(F_{x}, F_{\pi}\right)$ are inside in a stability triangle $A B C$ such that the output gap parameter is strictly negative $\left(F_{x}<0\right)$ and the inflation rule parameter is larger than one $\left(F_{\pi}>1-\left(\frac{1-\beta}{\kappa}\right) F_{x}>1\right.$ or $\left.p(1)>0\right)$, according to the Taylor principle.

Proof. Eigenvalues are both stable $\left(\left|\lambda_{1}\right|<1\right.$ and $\left.\left|\lambda_{2}\right|<1\right)$ if and only if $p(-1)>0$, $p(1)>0$ and $D<1$. The condition $p(1)>0$ provides the Taylor principle condition. The negative condition on the output gap Taylor rule parameter is obtained as follows. The maximal value of the output gap Taylor rule parameter is necessarily on one of the apexes of the stability triangle ABC which corresponds to intersections of $p(-1)>0, p(1)>0$ and $D<1$. It turns out that the values of this parameter for the three points defining the triangle are ordered as follows and that the largest one $F_{x}(A)$ is strictly negative:

$$
F_{x}(B)<F_{x}(C)<F_{x}(A)<0 .
$$

Because $\gamma>0, \kappa>0$ and $0<\beta \leq 1$, one has:

$$
\frac{-2-T(\mathbf{A})}{\gamma}<-\frac{T(\mathbf{A})}{\gamma}<\frac{2-T(\mathbf{A})}{\gamma}<0 .
$$

In particular, the largest value of the output gap Taylor rule parameter is negative $F_{x}(A)<0$ because $\gamma \kappa>0$ and $0<\beta \leq 1$ :

$$
\text { If } \gamma>0, \kappa>0 \text { and } 0<\beta \leq 1 \text { then } F_{x}(A)<0 \Leftrightarrow 2<T(\mathbf{A})=1+\frac{1+\gamma \kappa}{\beta} \text {. }
$$


Q.E.D.

Proposition 2. If $\gamma>0, \kappa>0$ and $0<\beta \leq 1$, then for policy rule parameters within the stability triangle $A B C\left(\left|\lambda_{1}\right|<1\right.$ and $\left|\lambda_{2}\right|<1$ if and only if $p(-1)>0, p(1)>0$ and $D<1)$, the trace and determinant of the closed-loop system are lower than in the case of the open-loop laissez-faire system, where $0<\lambda_{1}<1<\frac{1}{\lambda_{1} \beta}=\lambda_{2}: T(\mathbf{A}+\mathbf{B F})<T(\mathbf{A})$ and $D(\mathbf{A}+\mathbf{B F})<1 \leq D(\mathbf{A})=1 / \beta$.

Proof: As $F_{x}=\frac{T(\mathbf{A}+\mathbf{B F})-T(\mathbf{A})}{\gamma}<0$ in the stability triangle ABC, this implies that $T(\mathbf{A}+\mathbf{B F})<T(\mathbf{A})$. A condition of the stability triangle is that the product of eigenvalues is smaller than one: $D(\mathbf{A}+\mathbf{B F})<1$. By contrast, the open-loop system determinant is the inverse of the discount factor, which is at least equal to one: $1 \leq D(\mathbf{A})=1 / \beta$, hence $D(\mathbf{A}+\mathbf{B F})<1 \leq D(\mathbf{A})=1 / \beta$.

Q.E.D.

\subsection{Negative versus Positive Feedback}

The fact that a negative output gap Taylor rule parameter is a necessary condition for the closed-loop dynamic system to have two eigenvalues within the unit circle, requires further investigation. In this section, we explain how to distinguish negative feedback from positive feedback in the scalar case. We explain how the sign of the negative feedback policy rule parameter changes when the sign of the transmission mechanism changes. We are then able to explain that, because of the intertemporal substitution effect of the interest rate on consumption in the transmission mechanism, a negative output gap Taylor rule parameter corresponds to a negative feedback mechanism in the Taylor rule. By contrast, with a delayed cost-of-capital mechanism, a positive output gap Taylor rule parameter corresponds to a negative feedback mechanism of the Taylor rule.

Let us consider any first order scalar case transmission mechanism model and a feedback rule for a policy target $x_{t}$ and a policy instrument $i_{t}$ (first order "single input, single output", "SISO" case) with $A, B$ and $F$ real numbers:

$$
\begin{aligned}
& x_{t+1}=A x_{t}+B i_{t}: \text { Transmission mechanism; } i_{t}=F x_{t}: \text { Feedback rule } \\
& x_{t+1}=(A+B F) x_{t}: \text { Closed loop system; } x_{t+1}=A x_{t}: \text { open-loop system }(F=0) .
\end{aligned}
$$

The closed-loop system is obtained after substitution of the feedback rule. The openloop system (or "laissez-faire") corresponds to no policy intervention: the feedback rule parameter $F$ is equal to zero.

Definition 3: A negative feedback stabilization mechanism is defined as a set of stabilizing policy parameters such that the expectation of the gap of the target with respect to its long-run equilibrium value with policy intervention is smaller than the expectation of the gap of the target without intervention.

In the scalar case with a single input and a single output, the negative feedback stabilization mechanism is obtained by the following set of policy rule parameters $F$ :

$$
\{F \in \mathbb{R} \text {, with } F \text { such that }|A+B F|<\min (1,|A|)\} .
$$

A negative feedback mechanism implies that $|A+B F|<|A|$, the policy rule parameter decreases the eigenvalue of the closed-loop dynamic system with respect to the open-loop dynamic system. It accelerates convergence and decreases the endogenous persistence (auto-correlation $|A+B F|$ ) of the policy target $x_{t}$ with respect to its open-loop persistence measured by $|A|$. However, this may not lead to stabilization in 
the case where both $|A+B F|$ and $|A|$ are larger than one. In order to have negative feedback and stabilization, one needs the closed-loop eigenvalue to be below one in absolute value: $|A+B F|<1<|A|$. In this case, moving from negative feedback with stabilization to "laissez-faire" corresponds to a saddle-node bifurcation if $A$ is larger than $1,-1<A+B F<1<A$, and to a a flip bifurcation if $A$ is lower than -1 , $A<-1<A+B F<1$. In the case $|A+B F|<|A|<1$, we have a negative feedback mechanism, but there is no bifurcation because the open-loop system is already stable (its eigenvalue $|A|$ is in the unit circle).

Proposition 4. In the scalar case, for a positive open-loop auto-correlation of the policy target $A>0$, a change of sign of the marginal correlation $B$ of the current policy instrument $i_{t}$ on the future value of the policy target $x_{t+1}$ in the monetary policy transmission mechanism implies a change of sign of the negative feedback rule parameter $F$ : $B>0$ implies $F<0$ or $B<0$ implies $F>0$, because negative feedback implies that $B F<0$.

Proof.

$$
\begin{aligned}
&|A+B F|<A \Rightarrow-A<A+B F<A \Rightarrow-2 A<B F<0 . \\
& \text { If } B>0 \Rightarrow-2 \frac{A}{B}<F<0 \text { else if } B<0 \Rightarrow 0<F<-2 \frac{A}{B} .
\end{aligned}
$$

QED.

In other words, inspecting only of the sign of the parameter $F$ of the proportional feedback rule is not a proof of negative feedback mechanism, one needs to inspect the sign of the transmission mechanism parameter $B=$ and check that $B F<0$.

negative feedback implies bounded feedback rule parameters on both sides, and not only an asymmetric boundary condition such as the Taylor principle $\left(F_{\pi}>1\right)$. An excessive opposite feedback reaction would lead to a lower closed-loop eigenvalue than an opposite sign of the open-loop eigenvalue $A+B F<-A$ if $A>0$. It could lead to instability in the case where $A+B F<-1<-A$, if $A>0$.

When a loss function includes a quadratic cost on the volatility of the policy instrument, the reduced form of the optimal policy feedback rule parameter $F$ does not change the sign of the eigenvalue with respect to the open-loop eigenvalue. Else, it would lead to more volatility of the policy instrument for the same absolute value of the eigenvalue of the policy target. Hence, the range of variation of the policy instrument is such that $0 \leq A+B F<A$ and $-2 A<-A \leq B F<0$ (see Chatelain and Ralf (2016) for Ramsey optimal policy with a scalar case model). In this paper, the area of Ramsey optimal policy reduced form rule parameters remains on the same side of the center of the stability triangle (see Ramsey optimal policy section).

Proposition 5. If $\gamma>0, \kappa>0$ and $0<\beta \leq 1$, a negative Taylor rule parameter on the output gap $F_{x}<0$ is a necessary condition for a negative feedback mechanism in the New-Keynesian model.

Proof. The New-Keynesian transition matrix is:

$$
\left(\begin{array}{c}
E_{t} x_{t+1} \\
E_{t} \pi_{t+1}
\end{array}\right)=\left(\begin{array}{cc}
1+\frac{\gamma \kappa}{\beta}+\gamma F_{x} & -\frac{\gamma}{\beta}+\gamma F_{\pi} \\
-\frac{\kappa}{\beta} & \frac{1}{\beta}
\end{array}\right)\left(\begin{array}{c}
x_{t} \\
\pi_{t}
\end{array}\right) .
$$

Let us consider a particular initial output gap shock where $x_{0}>0$ and $\pi_{0}=0$. We consider this particular case, because we only seek a necessary condition in order to pro- 
vide an economic intuition explaining why the output gap rule parameter is negative for negative feedback. The necessary and sufficient conditions have been given in proposition 1: they define the stability triangle.

With the output gap shock where $x_{0}>0$ and $\pi_{0}=0$, the output equation corresponds to the scalar case. Negative feedback mechanism implies that the expectation of the output gap with policy intervention $E_{0} x_{1}$ should be lower than the expectation of next period's output gap without policy intervention $E_{0} x_{1, F_{x}=0}$ :

$$
E_{0} x_{1}=\left(1+\frac{\gamma \kappa}{\beta}+\gamma F_{x}\right) x_{0}<E_{0} x_{1, F_{x}=0}=\left(1+\frac{\gamma \kappa}{\beta}\right) x_{0} \Leftrightarrow \gamma F_{x}<0
$$

Since $\gamma>0$ this implies $F_{x}<0$. Because of the intertemporal substitution effect on consumption $B=\gamma>0$, a rise of the interest rate goes hand in hand with a rise of future output gap. Hence, negative feedback implies a negative output gap-Taylor-rule parameter $F=F_{x}<0$.

Q.E.D.

\subsection{Alternative Monetary Policy Transmission Mechanism}

This section seeks an alternative ad hoc monetary policy transmission mechanism such that negative feedback is compatible with a positive output gap rule parameter and a positive inflation Taylor rule parameter.

In the New-Keynesian model, the production function does not depend on capital. Hence, the monetary policy transmission channel of the cost of capital is assumed away. Central bankers often believe that, for a positive output gap, a rise of the interest rate leads to a fall of current output gap which leads to a fall of future inflation. For example, Taylor (1999) assumes no intertemporal substitution effect $(\gamma=0)$ but a negative effect of the user cost of capital on current output taking into account current inflation instead of expected inflation in the cost of capital. He also assumes an accelerationist Phillips curve effect, with an opposite negative correlation of future inflation with current output:

$$
x_{t}=-a\left(i_{t}-\pi_{t}\right) \text { and } \pi_{t+1}=\pi_{t}+b x_{t}=\pi_{t}-a b\left(i_{t}-\pi_{t}\right) \text {, with } a>0, b=-\kappa>0 .
$$

This dynamic system has only one dimension. It is similar to the extreme case of the New-Keynesian Phillips curve where all labor is financed by working capital so that $\kappa_{i}=a b>0$ (Christiano et al. (2010), Bratsiotis and Robinson (2016)). Future inflation is negatively correlated with the cost of working capital and not at all with the cost of labor. Because there is only one recursive dimension, the Taylor rule responds only to inflation: $i_{t}=F_{\pi} \pi_{t}$. The closed-loop system converges faster than the open-loop (negative feedback) if and only if the inflation Taylor rule parameter is larger than one (Taylor principle) and bounded from above:

$$
\begin{aligned}
-\pi_{t} & <\pi_{t+1}=\left(1+a b-a b F_{\pi}\right) \pi_{t}<\pi_{t} \Leftrightarrow-2-a b<-a b F_{\pi}<-a b \Leftrightarrow \\
1 & <F_{\pi}<1+\frac{2}{a b} .
\end{aligned}
$$

Now, let us introduce a second recursive dimension for the output gap besides inflation dynamics, namely an ad hoc delayed cost-of-capital effect. An increase of the interest rate 
leads to a decrease of future production $(\gamma<0)$. Based on microeconomic foundations assuming that some agents face credit rationing or limited asset market participation, this negative aggregate intertemporal elasticity of substitution is found in heterogeneous agents New-Keynesian models (Bilbiie (2008), Bilbiie and Straub (2013)). Empirically, Havranek (2015) and Havranek et al. (2015) found near zero estimates at macro level or negative estimates of $\gamma$ for some countries in their meta-analysis. Mavroeidis et al. (2014) found hundreds of negative estimates of $\kappa$ using USA data.

Proposition 6. For $0<\beta \leq 1$ and for the alternative transmission mechanism, $\gamma<$ $0, \kappa<0$, a necessary condition for having two stable eigenvalues $\left(\left|\lambda_{1}\right|<1\right.$ and $\left.\left|\lambda_{2}\right|<1\right)$ in the closed-loop transition matrix of the New-Keynesian model is that rule parameters $\left(F_{x}, F_{\pi}\right)$ are inside a stability triangle such that the output gap parameter is strictly positive $\left(F_{x}>0\right)$ and the inflation rule parameter is above one $\left(F_{\pi}>1-\left(\frac{1-\beta}{\kappa}\right) F_{x}\right)$, according to the Taylor principle.

Proof. Same computations as for Proposition 2 with opposite signs for $\gamma$ and $\kappa$.

The stability triangle in Figure 2 with opposite sign restrictions $\gamma<0$ and $\kappa<0$, is symmetric to the stability triangle of Figure 1 with respect to the horizontal axis in the positive quadrant of $\left(F_{x}, F_{\pi}\right)$. The economic intuition is that a rise of the interest rate today implies a fall of next period's output which implies a fall of inflation two-period from now. Then, negative feedback implies a positive output gap Taylor rule parameter.

\subsection{Determinacy with Forward-looking Interest Rate}

This section investigates why determinacy in the New-Keynesian model with a Taylor rule seeks two unstable eigenvalues and hence recommends a positive feedback mechanism for the central bank to stabilize the output gap and inflation.

Blanchard and Kahn's (1980) condition for the solution of a linear rational expectations dynamic system to be determinate (unique) is that the number of predetermined variables should be equal to the number of stable eigenvalues. In this paper, which variables are backward-looking (predetermined) and which are forward-looking, however, is an ad hoc choice of the researcher as it was the rule in ad hoc rational expectations models of the 1970s (Chatelain and Ralf (2019c)).

Blanchard and Kahn (1980) acknowledge that their determinacy condition is always satisfied for optimal growth and optimal control with a linear quadratic regulator when concavity conditions are satisfied. In this case, the ad hoc matrix of the linear rational expectations system is replaced by the symplectic matrix of a Hamiltonian system derived from optimal conditions with Lagrange multipliers. A symplectic matrix (its transpose is similar to its inverse) is such that the number of unstable eigenvalues is equal to the number of stable eigenvalues. The fact that a state variable or a costate variable is forward-looking or predetermined is endogenously derived from optimal control or the dynamic Stackelberg game program and its transversality conditions. The number of predetermined variables is equal to the number of forward-looking variables.

The New-Keynesian model is a hybrid model of optimal behavior for the private sector and of ad hoc behavior for the policy maker described by a policy instrument (the interest rate) which reacts to private sector variables according to an ad hoc feedback Taylor rule. The fact that the output gap and inflation are forward-looking is endogenously derived from the private sector's optimal control program. By contrast, the assumption that the interest rate and its lag are forward-looking variables is an ad hoc choice by a researcher. A researcher could as well select the opposite assumption that the interest and its lag 
are predetermined variables. It is only an arbitrary assumption as it was done in $a d$ hoc rational expectations models in the 1970s.

Using only Blanchard and Kahn's (1980) condition, it is not possible to prove that policy maker's instruments of feedback rules are necessarily forward-looking. To prove this would require a model of intertemporal optimal behavior by the policy maker. But, consistent with negative feedback mechanism, Ramsey optimal policy proves the opposite: policy instruments are backward-looking when policy targets are forward-looking.

In the New-Keynesian model, the two policy targets (inflation and output gap) are forward-looking variables, because they are costate variables of private sector optimal intertemporal behavior. In a second step, the New-Keynesian theory uses the ad hoc assumption that the policy instrument (interest rate) in the Taylor rule is a forwardlooking variable. As seen in the next section, Ramsey optimal policy implies something else: the policy instrument (interest rate) and its lag are backward-looking variables, because the two policy targets (inflation and output gap) are forward-looking variables.

Once it is assumed that the interest rate is forward-looking as well as inflation and the output gap, and once it is assumed that the predetermined variable of the private sector (wealth or public debt) is set to zero in all periods (Gali (2015), footnote 3), the model is a degenerate rational expectations model without predetermined variable. In this case, any shock leads to an instantaneous jump back to the long-run equilibrium value of inflation and output which is the only available anchor. Hence, there is no transitory dynamics. There is neither a need for stabilization policy nor for a feedback Taylor rule.

In order to introduce transitory dynamics, a New-Keynesian convention is to add ad $h o c$ autoregressive components to the cost-push shock and to the output gap shock. The expectations of these two autoregressive components provide two predetermined variables with transitory dynamics on which inflation and the output gap can be anchored. The fact that these anchors are not observable may be worrisome for central bank practitioners who usually anchor inflation on observable variables, such as their policy instruments.

Once the decision is taken by the researcher on which variables are forward-looking, Blanchard and Kahn's (1980) determinacy condition for ad hoc rational expectations linear systems is applied mechanically. The number of stable eigenvalues should be equal to the number of predetermined variables. There are two exogenous predetermined variables with their own stable eigenvalues corresponding to their auto-correlation parameters. There are three endogenous forward-looking variables (output gap, inflation and the interest rate) whose dynamics corresponds to a closed-loop endogenous system including a feedback Taylor rule in two dimensions. This implies that this two dimensional system should have two unstable eigenvalues. Hence, the determinacy area in the plane of the policy rule parameters corresponds to the areas where there are two unstable eigenvalues (regions 4.3 and 4.4 with $D>1$ and region 2 in Figure 1 ).

Because the Taylor rule parameters determine the stability or instability of eigenvalues, as a particular case of Wonham's (1967) pole placement theorem, when they force eigenvalues to be unstable instead of stable, they correspond to a positive feedback mechanism in the sense of control.

The determinacy area includes unlikely unbounded areas which are allowed by the positive feedback mechanism following the arbitrary assumption that the interest rate and its lag are forward-looking:

Region 2, first case: Negative unbounded output gap rule parameter $F_{x}<0$ but where the inflation rule parameter is positive but does not satisfy the Taylor principle is satisfied for the inflation rule parameter $0<F_{\pi}<1$. 
Region 2, second case: Negative unbounded output gap rule parameter $F_{x}<0$ and negative unbounded inflation rule parameter $F_{\pi}<0$.

Region 4, second case: Negative unbounded output gap rule parameter $F_{x}<0$ even though the Taylor principle is satisfied for unbounded large inflation rule parameter $F_{\pi}>1$.

Region 4, third case: Unlikely unbounded positive rule parameters $F_{\pi}>2, F_{x}>1$.

These guidelines suggest contradictory and unbounded values for Taylor rule parameters. They are hardly believable to policy-makers. Hence, New-Keynesian theory requires additional ad hoc boundary restrictions in order to remain in the subset of the determinacy region with plausible parameters $1<F_{\pi}<2$ and $0 \leq F_{x}<1$. This area is similar to the condition found in the Taylor model based on the cost of capital and negative feedback mechanism for the inflation Taylor rule parameter: $1<F_{\pi}<1+\frac{2}{a b}$. But the signs of the transmission mechanism on the output gap and of the feedback mechanism are the opposite.

\subsection{Determinacy with Predetermined Interest Rate}

Let us still assume a Taylor rule. But, as an alternative thought experiment, we can assume that, besides the two predetermined autoregressive forcing variables, the interest rate is arbitrarily predetermined as well as its lag, with given initial values $i_{0}$ and $i_{1}$ (or $i_{0}$ and $i_{-1}$ ). This assumption is no more or no less theoretically grounded than the alternative ad hoc assumption of the previous section. Then the model includes four predetermined variables. Blanchard and Kahn's (1980) determinacy condition implies that four eigenvalues should be stable. The endogenous two-dimensional system should be stable. This time, the determinacy area shifts to the stability triangle with negative feedback mechanism.

Once initial values of the interest rate are given, the problem of sunspot indeterminacy does not occur, because initial values of the forward-looking policy target are anchored on the two values of the interest rate by the Taylor rule (similar computations can be done for $i_{0}$ and $i_{-1}$ ):

$$
\begin{aligned}
& i_{0}=F_{x} x_{0}+F_{\pi} \pi_{0} \\
& i_{1}=E_{t} i_{1}=E_{t}\left(F_{x} x_{1}+F_{\pi} \pi_{1}\right)=\left(\begin{array}{cc}
F_{x} & F_{\pi}
\end{array}\right)\left(\begin{array}{cc}
1+\frac{\gamma \kappa}{\beta}+\gamma F_{x} & -\frac{\gamma}{\beta}+\gamma F_{\pi} \\
-\frac{\kappa}{\beta} & \frac{1}{\beta}
\end{array}\right)\left(\begin{array}{l}
x_{0} \\
\pi_{0}
\end{array}\right) \\
& i_{1}=\left(\begin{array}{ll}
F_{x}\left(\gamma F_{x}+\frac{\kappa}{\beta} \gamma+1\right)-\frac{\kappa}{\beta} F_{\pi} & \frac{1}{\beta} F_{\pi}+F_{x}\left(\gamma F_{\pi}-\frac{1}{\beta} \gamma\right.
\end{array}\right)\left(\begin{array}{l}
x_{0} \\
\pi_{0}
\end{array}\right) .
\end{aligned}
$$

One solves the linear system:

$$
\left(\begin{array}{c}
i_{1} \\
i_{0}
\end{array}\right)=\left(\begin{array}{cc}
F_{x}\left(\gamma F_{x}+\frac{\kappa}{\beta} \gamma+1\right)-\frac{\kappa}{\beta} F_{\pi} & \frac{1}{\beta} F_{\pi}+F_{x}\left(\gamma F_{\pi}-\frac{1}{\beta} \gamma\right) \\
F_{x} & F_{\pi}
\end{array}\right)\left(\begin{array}{l}
x_{0} \\
\pi_{0}
\end{array}\right) .
$$

Initial values of forward-looking variables are anchored as follows: 


$$
\left(\begin{array}{c}
x_{0} \\
\pi_{0}
\end{array}\right)=\left(\begin{array}{cc}
F_{x}\left(\gamma F_{x}+\frac{\kappa}{\beta} \gamma+1\right)-\frac{\kappa}{\beta} F_{\pi} & \frac{1}{\beta} F_{\pi}+F_{x}\left(\gamma F_{\pi}-\frac{1}{\beta} \gamma\right) \\
F_{x} & F_{\pi}
\end{array}\right)^{-1}\left(\begin{array}{c}
i_{1} \\
i_{0}
\end{array}\right) .
$$

They exclude sunspots although the equilibrium is a sink. The determinacy area is the bounded stability triangle represented in Figure 1.

Consider now Figure 2, with an alternative transmission mechanism: a delayed cost of capital and an accelerationist Phillips curve $\gamma<0, \kappa<0$. Assume that the interest rate and its lag are predetermined. The determinacy area is now a bounded triangle where the Taylor principle is satisfied for the inflation Taylor rule parameter and where the output gap Taylor rule parameter is positive.

By contrast, the determinacy area with the New-Keynesian transmission mechanism $\gamma>0, \kappa>0$ assuming the interest rate and its lag to be forward looking, includes a wide range of unbelievable unbounded areas, where Taylor rule parameters are too large and/or have negative signs.

\section{RAMSEY OPTIMAL POLICY}

For the New-Keynesian transmission mechanism of section 2, Gianonni and Woodford (2003) analyze Ramsey optimal policy in a model with an infinite time horizon. Kara (2007) computes Ramsey optimal policy with a probability to renege commitment in each period. The key change with respect to the infinite horizon model is that the discount factor is now multiplied by the probability of not reneging commitment $0<$ $q \leq 1$, so that the range of values of this "credibility augmented discount factor", $0<$ $\beta q \leq 1$, is much larger than for the discount factor alone: $0.98<\beta \leq 1$. Several names have been given to this kind of policy: "stochastic replanning" (Roberds, 1987) or "quasi commitment" (Schaumburg and Tambalotti, 2007, Kara 2007). The assumption is observationally equivalent to Chari and Kehoe's (1990) optimal policy under sustainable plans, facing a punishment threat at a given horizon in case of deviation from an optimal plan (Fujiwara, Kam, Sunakawa (2019)). The discretionary equilibrium where the central bank re-optimizes continuously without any credibility $(q=0)$ is a point of measure zero with respect to the continuous interval $q \in] 0,1]$. Since Ramsey optimal policy under quasi-commitment includes the case of very low credibility such as $q=10^{-7}$ the discretionary equilibrium is not a relevant equilibrium (Chatelain and Ralf (2018b)).

The contribution of this section is to use an intermediate step of Chatelain and Ralf's (2019a) algorithm in order to locate the full range of reduced form Taylor rule parameters of Ramsey optimal policy when varying the central bank preferences in the plane of Taylor rule parameters.

The policy maker minimizes the expectation of the present value $W$ of a discounted quadratic loss function $L_{t}$ over a finite horizon $T \geq 3$. He chooses the interest rate with respect to the policy targets (inflation and the output gap) with a positive weight on the second policy target (output gap) $\mu_{x} \geq 0$ and a weight normalized to one, $\mu_{\pi}=1$, for inflation (the limit case $\mu_{\pi}=0$ can also be taken into account). We have a strictly positive adjustment cost parameter $\mu_{i}>0$ on the volatility of the policy instrument (the interest rate) and a discount factor $\beta$ : 


$$
E(W)=-E_{t} \sum_{t=0}^{T} \beta^{t}\left\{\mu_{\pi} \frac{\pi_{t}^{2}}{2}+\mu_{x} \frac{x_{t}^{2}}{2}+\mu_{i} \frac{i_{t}^{2}}{2}\right\}, T \geq 3,
$$

subject to the private sector's New-Keynesian four equations model (equations (1) to (4)), with initial conditions for the predetermined state variables and natural boundary conditions for the private sector's forward variables. Denoting Lagrangian multipliers $\phi_{x, t}$ for the consumption Euler equation and $\phi_{\pi, t}$ for the New-Keynesian Phillips curve, the Lagrangian $\mathcal{L}$ is:

$$
\begin{aligned}
\mathcal{L}=-E_{0} \sum_{t=0}^{T} \beta^{t} & \left\{\mu_{\pi} \frac{\pi_{t}^{2}}{2}+\mu_{x} \frac{x_{t}^{2}}{2}+\mu_{i} \frac{i_{t}^{2}}{2}\right. \\
& \left.+\phi_{x, t}\left[x_{t}-x_{t+1}+\sigma\left(i_{t}-\pi_{t+1}\right)\right]+\phi_{\pi, t}\left[\pi_{t}-\beta_{t} \pi_{t+1}-\kappa x_{t}\right]\right\} .
\end{aligned}
$$

The law of iterated expectations has been used to eliminate the expectations that appeared in each constraint. Because of the certainty equivalence principle for determining optimal policy in the linear quadratic regulator including additive normal random shocks (Simon (1956)), the expectations of random variables $u_{t}$ are set to zero and do not appear in the Lagrangian.

Since inflation and the output gap are assumed to be forward-looking, they are optimally chosen at the initial date $t=0$ and at the final date $t=T$ according to transversality conditions, also called natural boundary conditions:

$$
\phi_{\pi, t=0}=\phi_{x, t=0}=\phi_{\pi, t=T}=\phi_{x, t=T}=0 \text { (Transversality conditions). }
$$

The Hamiltonian system with Lagrange multipliers of the linear quadratic regulator includes two stable roots and two unstable roots:

$$
\begin{aligned}
x_{t} & =x_{t+1}+\sigma\left(i_{t}-\pi_{t+1}\right) \\
\pi_{t} & =\beta_{t} \pi_{t+1}+\kappa x_{t} \\
\frac{\partial \mathcal{L}}{\partial \pi_{t}} & =0 \Rightarrow \mu_{\pi} \pi_{t}+\phi_{\pi, t}-\phi_{\pi, t-1}-\frac{\sigma}{\beta} \phi_{x, t-1}=0 \text { for } 0 \leq t \leq T, \\
\frac{\partial \mathcal{L}}{\partial x_{t}} & =0 \Rightarrow \mu_{x} x_{t}-\kappa \phi_{\pi, t}+\phi_{x, t}-\frac{1}{\beta} \phi_{x, t-1}=0 \text { for } 0 \leq t \leq T .
\end{aligned}
$$

A bounded optimal plan is a set of bounded processes $\left\{\pi_{t}, x_{t}, i_{t}, \phi_{x, t}, \phi_{\pi, t}\right\}$ for date $0 \leq$ $t \leq T$ of the Hamiltonian system that satisfy the four monetary transmission mechanism equations, the first order equations, and the optimal initial conditions.

The link between the Lagrange multipliers and the interest rate is given by:

$$
\frac{\partial \mathcal{L}}{\partial i_{t}}=0 \Rightarrow \mu_{i} i_{t}+\sigma \phi_{x, t}=0 \Rightarrow \phi_{x, t}=-\frac{\mu_{i}}{\sigma} i_{t} \text { for } 0 \leq t \leq T .
$$

The boundary conditions and the marginal conditions lead to the following constraints 
on the interest rate:

$$
\begin{aligned}
\frac{\partial \mathcal{L}}{\partial i_{t}} & =0 \Rightarrow \mu_{i} i_{t}+\sigma \phi_{x, t}=0 \Rightarrow \phi_{x, t}=-\frac{\mu_{i}}{\sigma} i_{t} \text { for } 0 \leq t \leq T \\
\phi_{\pi, t=0} & =\phi_{x, t=0}=\phi_{\pi, t=T}=\phi_{x, t=T}=0 \text { hence } \\
i_{-1} & =i_{-2}=0=i_{T-1}=i_{T-2}\left(i_{0} \neq 0 \text { for the shortest horizon } T=3\right) .
\end{aligned}
$$

Using the method of undetermined coefficients, Ljungqvist and Sargent (2012, chapter 19.3.1, step 1, p.769) solve a Riccati equation which allows to compute endogenous optimal negative feedback rule parameters $\left(F_{x, R}, F_{\pi, R}\right)$. Anderson, Hansen, McGrattan and Sargent (1996, p.203) consider that autoregressive shocks are initially set to zero $\left(\mathbf{z}_{0}=\mathbf{0}\right)$ to solve this Riccati equation. They do not depend on autoregressive parameters of exogenous variables. Anderson, Hansen, McGrattan and Sargent (1996, p.203) take into account the exogenous autoregressive process of forcing variables. They solve the rule parameter responding to shocks $F_{z, R}, F_{u, R}$. This step-one representation of the Ramsey optimal policy rule depends on all four variables of the private sector:

$$
i_{t}=F_{x} x_{t}+F_{\pi} \pi_{t}+F_{z} z_{t}+F_{u} u_{t} .
$$

Besides the two stable eigenvalues of the block of endogenous variables, there are the two stable eigenvalues that correspond to the autoregressive parameters of the block of the two predetermined and exogenous forcing variables (productivity and cost-push shock).

Ljungqvist and Sargent (2012, chapter 19, step 2) compute the linear stable subspace constraint between Lagrange multipliers (in this paper: $\phi_{x, t}, \phi_{\pi, t}$ ) and the variables of the private sector: $\phi_{t}=\mathbf{P}_{y} \mathbf{y}_{t}+\mathbf{P}_{z} \mathbf{z}_{t}$. The matrix $\mathbf{P}_{y}$ is the unique solution of a discretetime Riccati equation (Ljungqvist and Sargent (2012), chapter 19) and $\mathbf{P}_{z}$ is the unique solution of a Sylvester equation in the augmented discounted linear quadratic regulator (Anderson, Hansen, McGrattan and Sargent (1996), pp. 202-204).

Ljungqvist and Sargent (2012, chapter 19, step 3) substitute the forward-looking variables (in this paper: $x_{t}, \pi_{t}$ ) in the rule by their Lagrange multipliers (in this paper: $\left.\phi_{x, t}, \phi_{\pi, t}\right)$ using the linear constraint $\phi_{t}=\mathbf{P}_{y} \mathbf{y}_{t}+\mathbf{P}_{z} \mathbf{z}_{t}$. This representation of the Ramsey optimal policy rule is a function of four predetermined variables $\left(\phi_{t}, \mathbf{z}_{t}\right)$ which are not observable.

Ljungqvist and Sargent (2012, chapter 19, step 4) finally solve for the optimal initial anchor of forward-looking variables on predetermined variables:

$$
\phi_{t}=\mathbf{P}_{y} \mathbf{y}_{t}+\mathbf{P}_{z} \mathbf{z}_{t}, \phi_{0}=\mathbf{0}, \mathbf{P}_{\mathbf{y}} \text { invertible } \Longleftrightarrow \mathbf{y}_{0}=-\mathbf{P}_{y}^{-1} \mathbf{P}_{z} \mathbf{z}_{0} .
$$

Furthermore, Ljunqgvist and Sargent (2012, chapter 19.3.7) mention another representation of a Ramsey optimal policy rule which depends on lags of the policy instruments and predetermined variables.

In the article of Giannoni and Woodford (2003) a different Ramsey optimal policy rule which depends on lags of policy instruments and of forward-looking variables (inflation and the output gap) is presented. All variables are observable. The predetermined autoregressive forcing variables $\left(z_{t}, u_{t}\right)$ are substituted by a one period lag and a two period lag of the interest rate $\left(i_{t-1}, i_{t-2}\right)$. Chatelain and Ralf $(2018 \mathrm{c})$ demonstrate that the sum of parameters of the lags of funds rate is smaller than one for this representation of the solution of Ramsey optimal policy, so that Giannoni and Woodford (2003) "super- 
inertial" policy rules are ruled out. Other observationally equivalent representations of the Ramsey optimal policy rule may use leads or lags of other variables than the policy instrument.

All these various representations of Ramsey optimal policy rules correspond to linear substitutions of the variables which are taken as the set of basis vectors. They are all observationally equivalent taking into account the other equations of the stable solution of the Hamiltonian system. The eigenvalues of the Hamiltonian system are invariant to these changes of basis related to a change of representation of the rule of Ramsey optimal policy.

Step 1 representation of the rule of Ramsey optimal policy, with inflation and output gap as rule parameters (for $\mathbf{z}_{0}=\mathbf{0}$ ), corresponds to the representation of Figure 1 showing bifurcations and areas for stable and unstable eigenvalues. For each of the infinite number of other observationally equivalent representations of the rule of Ramsey optimal policy, including leads or lags and/or linear changes of variables (basis vectors) within the stable subspace of Ramsey optimal policy, a similar Figure can be drawn to highlight stability and bifurcations.

Proposition 7. The Taylor rule parameters on inflation and on the output gap in Ljunqgvist and Sargent's (2012, Chapter 19) step 1 representation are located in a subset of the stability triangle of Section 2, which is located on the left-hand side of the center of the stability triangle. This center corresponds to both eigenvalues equal to zero.

Proof. Ramsey optimal policy assumes that inflation and the output gap are forwardlooking and that the policy instrument (the interest rate) and its lag are predetermined. Two stable eigenvalues in the block of two endogenous variables of the New-Keynesian model are required to satisfy Blanchard and Kahn's (1980) determinacy condition. The reduced form Taylor rule parameters have to lie in the area where both eigenvalues are stable, that is, in the stability triangle.

Q.E.D.

The key principle is that negative feedback mechanism is stabilizing forward-looking variables, such as output and inflation, during a finite short horizon (the duration of a monetary policy regime), leaning against inflation and output gap spirals.

For a numerical example, Table 2 and Figure 3 provide the boundaries of the triangle of the linear quadratic regulator (LQR) reduced form Taylor rule parameters, obtained by a simulation grid, varying the weights in the loss function in three dimensions. The sides of the LQR triangle correspond to the cases where the central bank minimizes only the variance of inflation (inflation nutter) without taking into account the zero lower bound constraint on the policy interest rate $\left(\mu_{i}=10^{-7}>0\right)$, or minimizes only the variance of output gap without taking into account the zero lower bound $\left(\mu_{i}=10^{-7}>0\right)$, or seeks only maximal inertia of the policy rate $\left(\mu_{i} \rightarrow+\infty\right)$.

Table 2: Step 1: LQR rule parameters for $\kappa=0.1, \gamma=0.5, \beta=0.99, \rho_{z}=\rho_{u}=0.9$. 


\begin{tabular}{|l|l|l|l|l|l|l|l|l|l|}
\hline Minimize only: & $\mu_{\pi}$ & $\mu_{x}$ & $\mu_{i}$ & $\left|\lambda_{1}\right|$ & $\left|\lambda_{2}\right|$ & $F_{\pi}$ & $F_{x}$ & $F_{z}$ & $F_{u}$ \\
\hline Inflation & 1 & 0 & $10^{-7}$ & $7.10^{-5}$ & 0.006 & 21.21 & -3.92 & -2.01 & 39.5 \\
\hline Inflation output gap & 4 & 1 & $10^{-7}$ & $4.10^{-7}$ & 0.819 & 4.76 & -2.27 & -2.01 & 17.6 \\
\hline Inflation output gap & 1 & 1 & $10^{-7}$ & $4.10^{-7}$ & 0.905 & 3.03 & -2.10 & -2.01 & 12.8 \\
\hline Inflation output gap & $1 / 4$ & 1 & $10^{-7}$ & $4.10^{-7}$ & 0.951 & 2.10 & -2.01 & -2.01 & 8.90 \\
\hline Output gap & 0 & 1 & $10^{-7}$ & $4.10^{-7}$ & 0.995 & 1.21 & -1.92 & -2.01 & 2.95 \\
\hline Output gap interest & 0 & 4 & 1 & 0.348 & 0.953 & 1.70 & -1.31 & -2.21 & 6.78 \\
\hline Output gap interest & 0 & 1 & 1 & 0.541 & 0.918 & 1.83 & -0.98 & -2.43 & 7.38 \\
\hline Output gap interest & 0 & $1 / 4$ & 1 & 0.663 & 0.878 & 1.87 & -0.82 & -2.42 & 7.55 \\
\hline Interest rate & 0 & 0 & $1(+\infty)$ & 0.748 & 0.833 & 1.89 & -0.74 & -2.46 & 7.60 \\
\hline Inflation interest & $1 / 4$ & 0 & 1 & 0.784 & 0.784 & 1.99 & -0.77 & -2.43 & 7.85 \\
\hline Inflation interest & 1 & 0 & 1 & 0.772 & 0.772 & 2.22 & -0.83 & -2.37 & 8.45 \\
\hline Inflation interest & 4 & 0 & 1 & 0.742 & 0.742 & 2.82 & -0.98 & -2.26 & 9.95 \\
\hline
\end{tabular}

The LQR triangle is contained within the stability triangle, see Figure 3. A similar analysis can be made for the alternative monetary policy transmission mechanism with $\gamma<0$ and $\kappa<0$, see Figure 4 .

An example of finding the optimal initial anchor, step 4 in the Ljungqvist and Sargent approach is given in Table 3 . Here the columns below $x_{0}$ and below $\pi_{0}$ indicate the weights of $z_{0}$ and $u_{0}$ in the VAR.

Table 3: Step 4: optimal initial anchors, $\kappa=0.1, \gamma=0.5, \beta=0.99, \rho_{z, x}=\rho_{z, \pi}=0.9$.

\begin{tabular}{|l|l|l|l|l|l|}
\hline Minimize only: & $\mu_{\pi}$ & $\mu_{x}$ & $\mu_{i}$ & $x_{0}$ & $\pi_{0}$ \\
\hline Inflation & 1 & 0 & $10^{-7}$ & $10^{-4} z_{0}-10.1 u_{0}$ & $10^{-5} z_{0}+10^{-6} u_{0}$ \\
\hline Inflation output gap & 4 & 1 & $10^{-7}$ & $10^{-6} z_{0}-1.25 u_{0}$ & $10^{-8} z_{0}+3.14 u_{0}$ \\
\hline Inflation output gap & 1 & 1 & $10^{-7}$ & $10^{-6} z_{0}-0.49 u_{0}$ & $10^{-8} z_{0}+4.91 u_{0}$ \\
\hline Inflation output gap & $1 / 4$ & 1 & $10^{-7}$ & $10^{-6} z_{0}-0.16 u_{0}$ & $10^{-6} z_{0}+6.66 u_{0}$ \\
\hline Output gap & 0 & 1 & $10^{-7}$ & $10^{-6} z_{0}+10^{-6} u_{0}$ & $-10^{-6} z_{0}+9.61 u_{0}$ \\
\hline Output gap interest & 0 & 4 & 1 & $0.35 z_{0}+1.53 u_{0}$ & $-0.56 z_{0}+7.18 u_{0}$ \\
\hline Output gap interest & 0 & 1 & 1 & $0.58 z_{0}+2.52 u_{0}$ & $-0.79 z_{0}+6.20 u_{0}$ \\
\hline Output gap interest & 0 & $1 / 4$ & 1 & $0.72 z_{0}+3.13 u_{0}$ & $-0.92 z_{0}+5.63 u_{0}$ \\
\hline Interest rate & 0 & 0 & $1(+\infty)$ & $0.73 z_{0}+3.14 u_{0}$ & $-0.92 z_{0}+5.63 u_{0}$ \\
\hline Inflation interest & $1 / 4$ & 0 & 1 & $5.00 z_{0}-10.3 u_{0}$ & $0.71 z_{0}-0.04 u_{0}$ \\
\hline Inflation interest & 1 & 0 & 1 & $4.45 z_{0}-10.3 u_{0}$ & $0.61 z_{0}-0.03 u_{0}$ \\
\hline Inflation interest & 4 & 0 & 1 & $3.45 z_{0}-10.2 u_{0}$ & $0.42 z_{0}-0.02 u_{0}$ \\
\hline
\end{tabular}

This leads to the following observations:

- When the central bank is an inflation nutter, both eigenvalues are close to zero (point $\Omega$ in Figure 3 corresponds to the case when both eigenvalues are exactly equal to zero). To stabilize inflation in the second step of the transmission mechanism, one has to stabilize the output gap in the first step. The optimal anchor leads to an initial jump of inflation to zero, whereas the output gap jump is not zero.

- The lower side of the LQR triangle corresponds to the no-lower-bound constraint (no cost of changing the policy rate) and to changes of the relative weight on the output gap variance with respect to inflation variance. The lower the weight $\mu_{\pi}$ for a given weight $\mu_{x}$, the lower $F_{\pi}$, the response of the interest rate to inflation in the Taylor rule, the larger the initial jump of inflation, the lower the initial jump of the output gap.

- When the central bank is an output gap nutter, one eigenvalue is zero (related to output gap stabilization in next period based on the Euler consumption equation). 
However, the other eigenvalue tends to one when the weight of inflation is zero. There is no margin of errors on the inflation Taylor rule parameters with respect to the saddlenode bifurcation This second eigenvalue is related to the New-Keynesian Phillips curve and the autoregressive parameter of inflation, which only occurs in the second period of the monetary policy transmission mechanism.

- The upper left side of the LQR triangle (closest to the saddle-node bifurcation) corresponds to a weight $\mu_{\pi}=0$ for inflation and rising relative cost of changing the policy instruments with respect to the output gap weight. The lower the weight $\mu_{x}$ for a given weight on the policy instrument $\mu_{i}$, the higher $F_{\pi}$ the response of interest rate to inflation in the Taylor rule, the lower the initial jump of inflation, the larger the initial jump of the output gap. "Mostly inflation eigenvalue" decreases from 0.995 to 0.833. "Mostly output gap eigenvalue" increases from zero to 0.748 , see Table 2.

- When the central bank uses minimum energy control (it minimizes only the volatility of its policy instrument (or "input"): $\mu_{x}=\mu_{\pi}=0, \mu_{i}>0$ ), the eigenvalue 0.748 corresponds to the stable eigenvalue of laissez-faire matrix $\sqrt{\beta} \mathbf{A}_{y y}$ and the second stable eigenvalue $0.833=1 / 1.20$ is the inverse of the unstable eigenvalue 1.20 of the laissez-faire matrix $\sqrt{\beta} \mathbf{A}_{y y}$.

- The upper right side of the LQR triangle corresponds to zero weight on the output gap, and a relative increase of the weight of inflation with respect to the weight of the policy instrument. The higher the weight $\mu_{\pi}$ for a given weight on the policy instrument $\mu_{i}$, the inflation Taylor rule parameter rises from 1.89 to 21.2 (inflation nutter case). The inflation initial jump decreases toward zero.

\section{HOPF BIFURCATION}

As has been seen in the previous section, different emphasis of monetary policy on inflation, output gap, and the interest rate lead to different eigenvalues of the dynamic system and therefore to different stability properties. As a result, we can state the following proposition.

Proposition 8. Shifting from Ramsey optimal policy (where the interest rate and its lag are predetermined variables) to a New-Keynesian Taylor rule with plausible Taylor rule parameters (e.g., $1<F_{\pi}<2$ and $0<F_{x}<1$ ) (where the interest rate and its lag are forward-looking variables), when the Taylor principle is satisfied in both regimes, corresponds to a Hopf bifurcation (crossing $D=1$ for $T^{2}-4 D<0$ ) in the New-Keynesian model.

\section{Proof.}

The interest rate and its lag are optimally predetermined in a regime of Ramsey optimal policy, whereas they are arbitrarily assumed to be forward-looking in the determinate solution of the New-Keynesian model with a Taylor rule.

For Ramsey optimal policy, the number of predetermined variables is equal to four when the two predetermined forcing variables are taken into account. In the case of determinacy in the new Keynesian model with a Taylor rule, this number is reduced to two, namely only the two forcing variables.

This implies that the controllable part of the New-Keynesian model includes two stable eigenvalues with Ramsey optimal policy. The policy rule parameters lie in the stability triangle ABC bounded by $p(1)>0, p(-1)>0$ and $D<1$, regions 4.1 and 4.2 in Figure 1. See also Figure 5 for a detailed view of a smaller range of possible parameter 
values.

By contrast, in the New-Keynesian model we have no stable eigenvalue. The determinacy regions are contained in regions 4.3 and 4.4 with $p(1)<0$ and $p(-1)<0$ either with conjugate complex or real eigenvalues larger than one in absolute values. As a plausible area for Taylor rule parameters $1<F_{\pi}<2$ and $0<F_{x}<1$ are given, see purple area in Figure 5.

Because both policy rules are in region 4 above saddle-node and flip bifurcation limits, defined by $p(1)>0$ and $p(-1)>0$, when modelers shift their theory on central bank behavior from Ramsey policy to New-Keynesian determinacy with Taylor rule they move from the red area inside the stablity triangle to the purple area outside the triangle, see Figure 5. Their change of theory corresponds to a Hopf bifurcation, crossing the Hopf bifurcation border $D=1$, line AB in Figures 1 and 5 .

Q.E.D.

\section{CHANGING THE HYPOTHESES ON SHOCKS}

The zero variance of shocks covers two factors: the variance of the random i.i.d. component $\varepsilon_{z, t}$ and the auto-correlation $\rho$ specific to forcing variables. In what follows we will briefly discuss how changes of these hypotheses affect the results.

Zero variance of the i.i.d. components $\varepsilon_{z, t}$ and $\varepsilon_{u, t}$ with a non-zero autocorrelation of forcing variables

For the New-Keynesian Taylor rule, this does not change the results because inflation and output gap can be anchored on deterministic autoregressive forcing variables.

For Ramsey optimal policy, this does not change the results, because the optimal endogenous policy rule parameters do not depend on the variance of shocks in the case of the augmented linear quadratic regulator. This is the certainty equivalent principle in the case of quadratic optimization subject to a linear dynamic system (Simon (1956)).

Zero autocorrelation of both forcing variables $z_{t}$ and $u_{t}$, with a non-zero variance of the i.i.d. components $\varepsilon_{z, t}$ and $\varepsilon_{u, t}$.

For the New-Keynesian Taylor rule, there are two forward-looking variables that are not predetermined. We then get a degenerate rational expectations equilibrium. As soon as shocks $\varepsilon_{z, t}$ and $\varepsilon_{u, t}$ are known, inflation and output gap instantaneously jump back to their respective long-run steady-state value, which is the only anchor available. This implies that the interest rate never deviates from its long-run steady-state value. The Taylor rule cannot be estimated. The long run steady state for output gap and inflation is a source for the Taylor rule parameters chosen in the New-Keynesian determinacy area (region 4 with $D>1$ and region 2 ).

For Ramsey optimal policy, there are two predetermined Lagrange multipliers set to zero, which optimally predetermine the interest rate and its lag. The optimal anchors of inflation and the output gap at the initial date (stacked in the vector $\mathbf{y}_{0}$ ) are given by $\mathbf{y}_{0}=-\mathbf{P}_{y}^{-1} \mathbf{P}_{z} \mathbf{z}_{0}$, where $\mathbf{z}_{0}$ corresponds to the expectations of both autoregressive forcing variables. With zero auto-correlation, this expectation is equal to zero for both shocks. Hence, $\mathbf{z}_{0}=0=\mathbf{y}_{0}$. The optimal initial anchor is also the long run steady state value of inflation and the output gap. The optimal interest rate never deviates from its long run steady state value: this is how it is predetermined at the initial date. The reduced form Taylor rule parameters for inflation and the output gap are different from zero $\left(\mathbf{F}_{y} \neq 0\right)$. But if both auto-correlation coefficients are equal to zero, $\rho_{i}=0 \Rightarrow \mathbf{F}_{z}=0$, the interest 
rate does not respond to non-autoregressive shocks. This is consistent with Simon's (1956) certainty equivalence principle. The long run steady state for output gap and inflation is a sink for the Taylor rule parameters chosen in the Ramsey optimal policy determinacy area (region 4 with $D<1$ ). This equilibrium corresponds to degenerate Ramsey optimal policy.

\section{Zero auto-correlation of only one of the forcing variables $\left(u_{t}\right)$.}

For the New-Keynesian Taylor rule, there are two forward-looking forcing variables for one predetermined forcing variable. For all periods, both, inflation and the output gap, are linear functions of $z_{t}: \pi_{t}=N_{\pi} z_{t}$ and $x_{t}=N_{x} z_{t}$. Hence, the output gap is a linear function of inflation $x_{t}=N_{x} N_{\pi}^{-1} \pi_{t}$. The recursive dynamic system boils down to a dynamic system of dimension one. The feedback rule needs to respond to only one linear combination of the two policy targets. This implies selecting one linear identification restriction for the Taylor rule parameters among an infinity of possibilities: $F_{x}=\alpha F_{\pi}$ with $\alpha \in \mathbb{R}$ or $F_{\pi}=0$. For $F_{\pi}=0$ and $F_{x} \neq 0$, the Taylor rule responds only to the output gap $\left(i_{t}=F_{x} x_{t}\right)$ which seemingly contradicts inflation targeting. It is observationally equivalent to another policy rule $i_{t}=F_{x} N_{x} N_{\pi}^{-1} \pi_{t}$, where we define $F_{\pi, 2}=F_{x} N_{x} N_{\pi}^{-1} \neq 0$ and $F_{x, 2}=0$, which describes "inflation targeting" and seemingly contradicts an output gap stabilization objective. Assuming a unique predetermined variable for two forwardlooking variables forces the coincidence that both forward-looking variables are exactly collinear. This assumption cannot "demonstrate" inflation targeting and deny an output gap stabilization objective, because they are exactly equivalent. The determinacy area for Taylor rule parameters is unchanged (region 4 with $D>1$ and region 2), but the chosen identification restriction has to be taken into account .

For Ramsey optimal policy, additional to the two forward-looking variables for one predetermined forcing variable there are two backward-looking Lagrange multipliers on inflation and on the output gap set to zero at the initial date. At the date of the initial optimization, both, initial inflation and initial output gap, are anchored as linear functions to the initial value of the unique forcing variable $z_{0}$, according to the formula: $\mathbf{y}_{0}=$ $-\mathbf{P}_{y}^{-1} P_{z} z_{0}$. Hence, at the initial date, the output gap is a linear function of inflation. But, during all the periods where the central bank does not re-optimize, the recursive dynamic system boils down to a dynamic system of dimension three. The feedback rule responds to output gap and inflation with unchanged formula for $\mathbf{F}_{y}$. The determinacy area for Ramsey optimal policy is unchanged (region 4 with $D<1$ ). Since the autocorrelation coefficient is now zero, the feedback rule responds only to the shock $z_{t}$ with a parameter $F_{z}$ found by solving a scalar Sylvester equation. The identification restriction is then $F_{u}=0$, which is implied by $\rho_{u}=0$ in a Sylvester equation of the augmented linear quadratic regulator.

\section{CONCLUSION}

In this paper we derived the dynamic properties of New-Keynesian Taylor rule policy and Ramsey optimal policy. Stability, determinacy, and anchors of policy variables were compared. According to the choice of the policy parameters, namely, the Taylor rule parameters corresponding to the inflation and the output target, the steady state can be a source or a sink with real or complex eigenvalues. Changing the policy doctrine and as a consequence the policy parameters, may change the stability properties, and bifurcations are possible. Moving in particular from Ramsey optimal policy to a New-Keynesian 
Taylor rule can be interpreted as a Hopf bifurcation.

Ramsey optimal policy can also take into account monetary and fiscal policy (Cardani et al. (2018), Gomis-Porqueras and Zhang (2019), Chatelain and Ralf (2019d, 2019e)). Further research may test these models, following Chatelain and Ralf (2017) tests and estimations in the case where the transmission mechanism is only the New-Keynesian Phillips curve. A key insight is that positive feedback stabilization policy requires more structural parameters to fit the data than negative feedback stabilization policy (Chatelain and Ralf (2018a)). This may lead to parameter identification issue (Chatelain and Ralf (2014)).

\section{References}

[1] Ackermann, J. (1972). Der Entwurf linearer Regelungssysteme im Zustandsraum. Regelungstechnik, 7, 297-300.

[2] Anderson E.W., Hansen L.P., McGrattan E.R. and Sargent T.J. (1996). Mechanics of Forming and Estimating Dynamic Linear Economies. in Amman H.M., Kendrick D.A. and Rust J. (editors) Handbook of Computational Economics, Elsevier, Amsterdam, 171-252.

[3] Aström K.J. \& Kumar, P. R. (2014). Control: A perspective. Automatica, 50, pp. $3-43$.

[4] Azariadis, C. (1993). Intertemporal macroeconomics, Blackwell Publishers, Oxford.

[5] Ball, L. (1994). Credible disinflation with staggered price-setting. The American Economic Review, 84, pp. 282-289.

[6] Barnett W.A. and Duzhak E.A. (2008). Non-Robust Dynamic Inferences from Macroeconometric Models: Bifurcation stratification of Confidence Region. Physica $A, 387$, pp. 3817-3825.

[7] Barnett W.A. and Duzhak E.A. (2010). Empirical Assessment of Bifurcations Regions within New-Keynesian models. Economic Theory, 45, pp. 99-128.

[8] Barnett W.A. and Chen G. (2015). Bifurcation of Macroeconometric Models and Robustness of Dynamical Inferences. Foundations and Trends®in Econometrics, 8, pp. 1-144.

[9] Benati L. and Goodhart C. (2010). Monetary Policy Regimes and Economic Performance: The historical record 1979-2008. In Handbook of Monetary Economics, vol 3, Friedman B.M. and Woodford M. editors, Elsevier B.V., pp. 1159-1236.

[10] Blanchard O.J. and Kahn C. (1980). The solution of linear difference models under rational expectations. Econometrica, 48, pp. 1305-1311.

[11] Bilbiie F.O. (2008). Limited asset market participation, monetary policy and (inverted) aggregate demand logic. Journal of Economic Theory, 140, pp. 162-196.

[12] Bilbiie F.O. and Straub R. (2013). Asset Market Participation, Monetary Policy Rules, and the Great Inflation. Review of Economics and Statistics, 95, pp. 377-392. 
[13] Bratsiotis, G. J. and Robinson, W. A. (2016). Unit Total Costs: An Alternative Marginal Cost Proxy for Inflation Dynamics. Macroeconomic Dynamics, 20, pp. 124 .

[14] Cardani, R., L. Menna, L. and P. Tirelli (2018). The optimal policy mix to achieve public debt consolidation. Macroeconomic Dynamics, 1-17.

[15] Chari, V. V., \& Kehoe, P. J. (1990). Sustainable plans. Journal of political economy, 98, pp. $783-802$.

[16] Chatelain, J. B. and K. Ralf (2014) Peut-on identifier les politiques économiques stabilisant une économie instable? Revue française d'économie, 29, pp. 143-178.

[17] Chatelain J.B. and Ralf K. (2016) Countercyclical versus Procyclical Taylor Principles. SSRN working paper.

[18] Chatelain J.B. and Ralf K. (2017) Can we Identify the Fed's Preferences? PSE working paper.

[19] Chatelain J.B. and K. Ralf (2018a) Publish and Perish: Creative Destruction and Macroeconomic Theory. History of Economic Ideas, 27, pp. 65-101.

[20] Chatelain, J. B. and K. Ralf (2018b) Imperfect Credibility versus No Credibility of Optimal Monetary Policy. PSE working paper.

[21] Chatelain, J. B. and K. Ralf (2018c) Super-inertial interest rate rules are not solutions of Ramsey optimal monetary policy. PSE working paper.

[22] Chatelain J.B. and K. Ralf (2019a) A Simple Algorithm for Solving Ramsey Optimal Policy with Exogenous Forcing Variables. Economics Bulletin 39(4), 2429-2440.

[23] Chatelain, J. B. and K. Ralf (2019b) How Macroeconomists Lost Control: Towards Dark Ages. Research Gate working paper.

[24] Chatelain, J. B. and K. Ralf (2019c) Policy Maker's Credibility with Predetermined Instruments for Forward-Looking Targets. PSE working paper.

[25] Chatelain, J. B. and K. Ralf (2019d) Ramsey Optimal Policy versus Multiple Equilibria with Fiscal and Monetary Interactions. PSE working paper.

[26] Chatelain, J. B. and K. Ralf (2019e) Ramsey Optimal Policy in the New-Keynesian Model with Public Debt. PSE working paper.

[27] Christiano, L. J., Trabandt, M., \& Walentin, K. (2010). DSGE models for monetary policy analysis. In Handbook of Monetary Economics, vol 3, Friedman B.M. and Woodford M. editors, Elsevier B.V., pp. 285-368.

[28] Cochrane J.H. (2011). Determinacy and identification with Taylor Rules. Journal of Political Economy, 119, pp. 565-615.

[29] Currie, D., \& Levine, P. (1985). Macroeconomic policy design in an interdependent world. In International Economic Policy Coordination, pp. 228-273. NBER. Cambridge University Press. 
[30] Doyle J.C. (1978). Guaranteed Margins for LQG Regulators. IIIE Transactions on Automatic Control. AC-23, pp. 756-757.

[31] Freiling, G. (2002). A survey of nonsymmetric Riccati equations. Linear algebra and its applications, 351, pp. 243-270.

[32] Fujiwara, I., Kam, T., \& Sunakawa, T. (2019). On two notions of imperfect credibility in optimal monetary policies. Economics Letters, 174, pp. 22-25.

[33] Gali J. (2015). Monetary Policy, Inflation and the Business Cycle. 2nd. edition, Princeton University Press, Princeton.

[34] Giannoni, M. P. \& Woodford M. (2003). How forward-looking is optimal monetary policy? Journal of Money, Credit, and Banking, 35, pp. 1425-1469.

[35] Giordani P. and Söderlind P. (2004). Solutions of macromodels with Hansen-Sargent robust policies: some extensions. Journal of Economics Dynamics and Control, 28, pp. 2367-2397.

[36] Gomis-Porqueras, P., \& Zhang, C. (2019). Optimal monetary and fiscal policy in a currency union with frictional goods markets. Macroeconomic Dynamics, 1-29

[37] Hall, R. E. (1988). Intertemporal substitution in consumption. Journal of political economy, 96, pp. 339-357.

[38] Hansen L.P. and Sargent T. (2008). Robustness, Princeton University Press, Princeton.

[39] Hansen L.P. and Sargent T. (2011). Wanting Robustness in Macroeconomics. In Handbook of Monetary Economics, vol 3(B), Friedman B.M. and Woodford M. editors, Elsevier B.V., pp. 1097-1155.

[40] Havranek T., Horvath R., Irsova Z. and Ruznak M. (2015). Cross-country heterogeneity in intertemporal substitution. Journal of International Economics, 96, pp. 100-118.

[41] Havránek, T. (2015). Measuring intertemporal substitution: The importance of method choices and selective reporting. Journal of the European Economic Association, 13, pp. 1180-1204.

[42] Kalman R.E. (1960). Contributions to the Theory of Optimal Control. Boletin de la Sociedad Matematica Mexicana, 5, pp. 102-109.

[43] Kara, H. (2007). Monetary policy under imperfect commitment: Reconciling theory with evidence. International Journal of Central Banking, 3, pp. 149-177.

[44] Leeper, E. M. (1991). Equilibria under 'active'and 'passive'monetary and fiscal policies. Journal of monetary Economics, 27, pp. 129-147.

[45] Ljungqvist L. and Sargent T.J. (2012). Recursive Macroeconomic Theory. 3rd edition. The MIT Press. Cambridge, Massaschussets. 
[46] Mavroeidis S., Plagbord-Moller M. and Stock J. (2014). Empirical Evidence of Inflation Expectations in the New-Keynesian Phillips Curve. Journal of Economic Literature, 52, pp. 124-188.

[47] Roberds, W. (1987). Models of policy under stochastic replanning. International Economic Review, 28, pp. 731-755.

[48] Romer D. (2012). Advanced Macroeconomics. 4th edition. McGraw-Hill.

[49] Schaumburg, E., and Tambalotti, A. (2007). An investigation of the gains from commitment in monetary policy. Journal of Monetary Economics, 54, pp. 302-324.

[50] Söderlind P. (1999). Solution and Estimation of RE Macromodels with Optimal Policy. European Economic Review, 43, pp. 813-23.

[51] Simaan, M., \& Cruz, J. B. (1973). Additional aspects of the Stackelberg strategy in nonzero-sum games. Journal of Optimization Theory and Applications, 11, pp. 613-626.

[52] Simon H.A. (1956). Dynamic Programming under Uncertainty with a Quadratic Criterion Function. Econometrica, 24, pp. 74-81.

[53] Sims, C. A. (2010). Rational inattention and monetary economics. In Handbook of Monetary Economics, vol 3, Friedman B.M. and Woodford M. editors, Elsevier B.V., pp. 155-181.

[54] Taylor J.B. (1999). The Robustness and Efficiency of Monetary Policy Rules as Guidelines for Interest Rate Setting by the European Central Bank. Journal of Monetary Economics. 43, pp. 655-679.

[55] Tinbergen J. (1952). On the theory of economic policy. North Holland.

[56] Wonham W.N. (1967). On pole assignment in multi-input controllable linear system. IEEE transactions on automatic control. 12, pp. 660-665.

[57] Zhou K, Doyle D.C. and Glover K. (1995). Robust and Optimal Control. Prentice Hall, New Jersey.

\section{APPENDIX A: Scilab code}

Download the open source software Scilab and copy and paste the following code in the command window, for given preferences of the central bank (Qpi, Qx, R) and given monetary policy transmission mechanism parameters (beta1, gamma1, kappa, rho1, rho2). Transition matrix is multiplied by $\sqrt{\beta}$ in order to take into account discounting as proposed by Anderson, Hansen, McGrattan and Sargent (1996). Formulas for Riccati, Sylvester and rule parameters are taken in Anderson, Hansen, McGrattan and Sargent (1996).

$\mathrm{Qpi}=4 ; \mathrm{Qx}=0 ; \mathrm{R}=1 ; \mathrm{Qxpi}=0$;

beta1 $=0.99$; gamma1 $=0.5 ;$ kappa $=0.1$;

rho1 $=0.9 ;$ rho $2=0.9 ;$ rho12 $=0$;

Qxrho1=0; Qpirho1=0; 


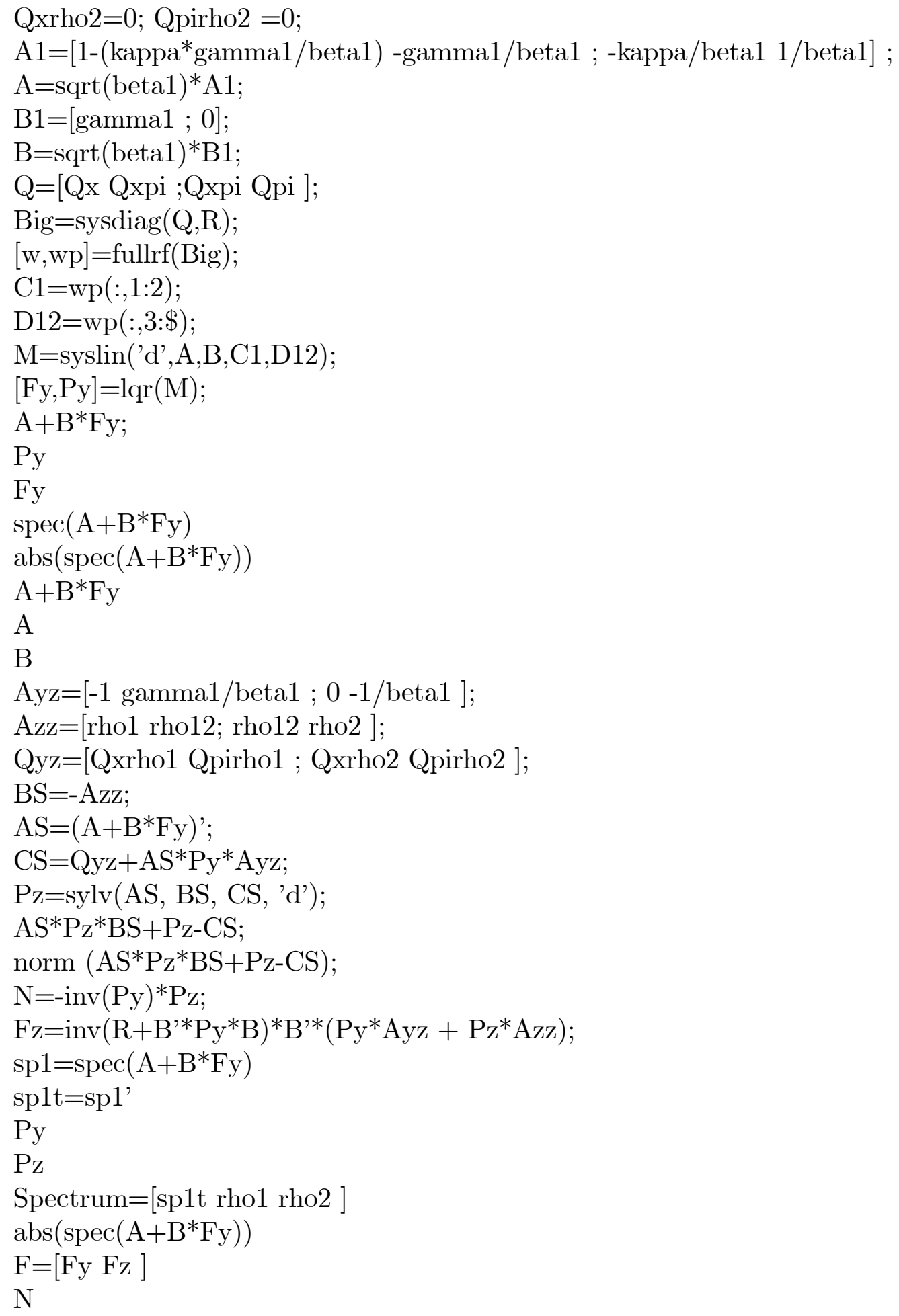

\section{APPENDIX B: Wonham theorem and pole placement}

Let $\mathbf{A}$ and $\mathbf{B}$ be real matrices of dimension $n \times n$ and $n \times m$ respectively. Let $\Lambda=$ $\left\{\lambda_{1}, \ldots, \lambda_{n}\right\}$ be an arbitrary set of $n$ complex numbers $\lambda_{i}$ such that any $\lambda_{i}$ with $\operatorname{Im}\left(\lambda_{i}\right) \neq 0$ appears in $\Lambda$ in a conjugate pair. 
Wonham (1967) pole placement theorem. The pair (A,B) is controllable $\left(\operatorname{rank}\left(\mathbf{B}, \mathbf{A B}, \mathbf{A}^{2} \mathbf{B}, \ldots, \mathbf{A}^{n-1} \mathbf{B}\right)=n\right)$ if and only if, for every choice of the set $\Lambda=$ $\left\{\lambda_{1}, \ldots, \lambda_{n}\right\}$, there is a matrix $\mathbf{F}$ such that $\mathbf{A}+\mathbf{B F}$ has $\Lambda$ for its sets of eigenvalues.

Wonham's (1967) pole placement theorem states that linear feedback rule parameters are always bifurcation parameters of controllable linear systems. Close to bifurcations limit values, a small change of the policy rule parameters leads to big qualitative change of the dynamics of the system to be controlled, as eigenvalues shift from being outside the unit circle to inside the unit circle.

\section{Pole placement using the canonical form.}

The characteristic polynomial for the open-loop eigenvalues and the desired closedloop eigenvalues corresponds to distinct coefficients (the trace and determinant):

$$
\lambda^{2}-T(\mathbf{A}) \lambda+D(\mathbf{A})=0 \text { and } \lambda^{2}-T(\mathbf{A}+\mathbf{B F}) \lambda+D(\mathbf{A}+\mathbf{B F})=0 .
$$

The canonical form of the dynamic system is such that:

$$
\widehat{A}=\left(\begin{array}{cc}
T(\mathbf{A}) & -D(\mathbf{A}) \\
1 & 0
\end{array}\right), \widehat{B}=\left(\begin{array}{l}
1 \\
0
\end{array}\right) .
$$

Hence, the closed-loop canonical model has the property that the feedback rule parameters appear only on the first line. It should correspond to given closed-loop trace and determinant.

$$
\widehat{A}+\widehat{B} \widehat{F}=\left(\begin{array}{cc}
T(\mathbf{A})+\widehat{F}_{x} & -D(\mathbf{A})+\widehat{F}_{\pi} \\
1 & 0
\end{array}\right)=\left(\begin{array}{cc}
T(\mathbf{A}+\mathbf{B F}) & -D(\mathbf{A}+\mathbf{B F}) \\
1 & 0
\end{array}\right)
$$

Then:

$$
\widehat{F}=\left(\begin{array}{cc}
\widehat{F}_{x} & \widehat{F}_{\pi}
\end{array}\right)=(T(\mathbf{A}+\mathbf{B F})-T(\mathbf{A})-(D(\mathbf{A}+\mathbf{B F})-D(\mathbf{A}))) .
$$

The feedback rule is equal to $\widehat{\mathbf{F}}$ times a similarity matrix which is the product of the canonical controllability matrix of $\widehat{\mathbf{A}}+\widehat{\mathbf{B}} \widehat{\mathbf{F}}$ and the inverse of the controllability matrix of $\mathbf{A}+\mathbf{B F}$ :

$$
\begin{aligned}
& \mathbf{F}=-\left(\begin{array}{cc}
T(\mathbf{A})-T & D-D(\mathbf{A}))
\end{array}\left(\begin{array}{cc}
1 & T(\mathbf{A}) \\
0 & 1
\end{array}\right)\left(\begin{array}{cc}
\gamma & \gamma\left(T(\mathbf{A})-\frac{1}{\beta}\right) \\
0 & -\frac{\kappa \gamma}{\beta}
\end{array}\right)^{-1}\right.
\end{aligned}
$$

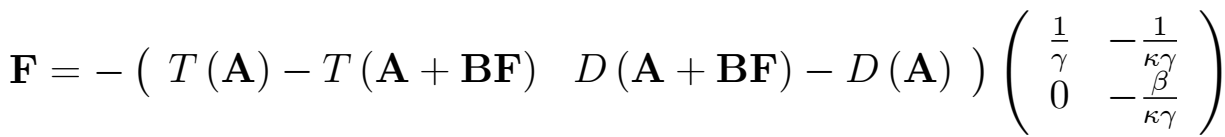

$$
\begin{aligned}
& \mathbf{F}=\left(\frac{1}{\gamma}(T(\mathbf{A}+\mathbf{B F})-T(\mathbf{A}))-\frac{1}{\gamma \kappa}(T(\mathbf{A}+\mathbf{B F})-T(\mathbf{A}))+\frac{\beta}{\gamma \kappa}(D(\mathbf{A}+\mathbf{B F})-D(\mathbf{A}))\right) .
\end{aligned}
$$

Pole placement using Ackermann's (1972) formula for a controllable system (we use the notation $\mathbf{A}+\mathbf{B F}$ instead of $\mathbf{A}-\mathbf{B F}$, hence the minus sign at the beginning):

$$
\mathbf{F}=-\left(\begin{array}{ll}
0 & 1
\end{array}\right)\left(\begin{array}{ll}
\mathbf{B} & \mathbf{A B}
\end{array}\right)^{-1}\left(\mathbf{A}^{2}-T(\mathbf{A}+\mathbf{B F}) \mathbf{A}+D(\mathbf{A}+\mathbf{B F})\right) .
$$

Applied on the New-Keynesian model: 


$$
\begin{aligned}
& \left.\mathbf{F}=-\left(\begin{array}{ll}
0 & 1
\end{array}\right)\left(\begin{array}{cc}
\gamma & \gamma\left(\frac{\kappa}{\beta} \gamma+1\right. \\
0 & -\frac{\kappa}{\beta} \gamma
\end{array}\right)\right)^{-1}\left(\left(\begin{array}{cc}
1+\frac{\gamma \kappa}{\beta} & -\frac{\gamma}{\beta} \\
-\frac{\kappa}{\beta} & \frac{1}{\beta}
\end{array}\right)^{2}-T\left(\begin{array}{cc}
1+\frac{\gamma \kappa}{\beta} & -\frac{\gamma}{\beta} \\
-\frac{\kappa}{\beta} & \frac{1}{\beta}
\end{array}\right)+D\left(\begin{array}{ll}
1 & 0 \\
0 & 1
\end{array}\right)\right) \\
& \mathbf{F}=\left(\frac{1}{\gamma}(T(\mathbf{A}+\mathbf{B F})-T(\mathbf{A}))-\frac{1}{\gamma \kappa}(T(\mathbf{A}+\mathbf{B F})-T(\mathbf{A}))+\frac{\beta}{\gamma \kappa}(D(\mathbf{A}+\mathbf{B F})-D(\mathbf{A}))\right) .
\end{aligned}
$$

The transfer function of the New-Keynesian model is:

$$
C(s I-A)^{-1} B=-\left(\begin{array}{ll}
1 & 1
\end{array}\right)\left(\begin{array}{cc}
1+\frac{\gamma \kappa}{\beta}-s & -\frac{\gamma}{\beta} \\
-\frac{\kappa}{\beta} & \frac{1}{\beta}-s
\end{array}\right)^{-1}\left(\begin{array}{l}
\gamma \\
0
\end{array}\right)=\frac{\gamma s-\frac{\gamma+\gamma \kappa}{\beta}}{s^{2}-T_{A} s+D_{A}} .
$$


Figure 1: Stability triangle in the plane of policy parameters, $\gamma=0.5, \kappa=0.1, \beta=0.99$

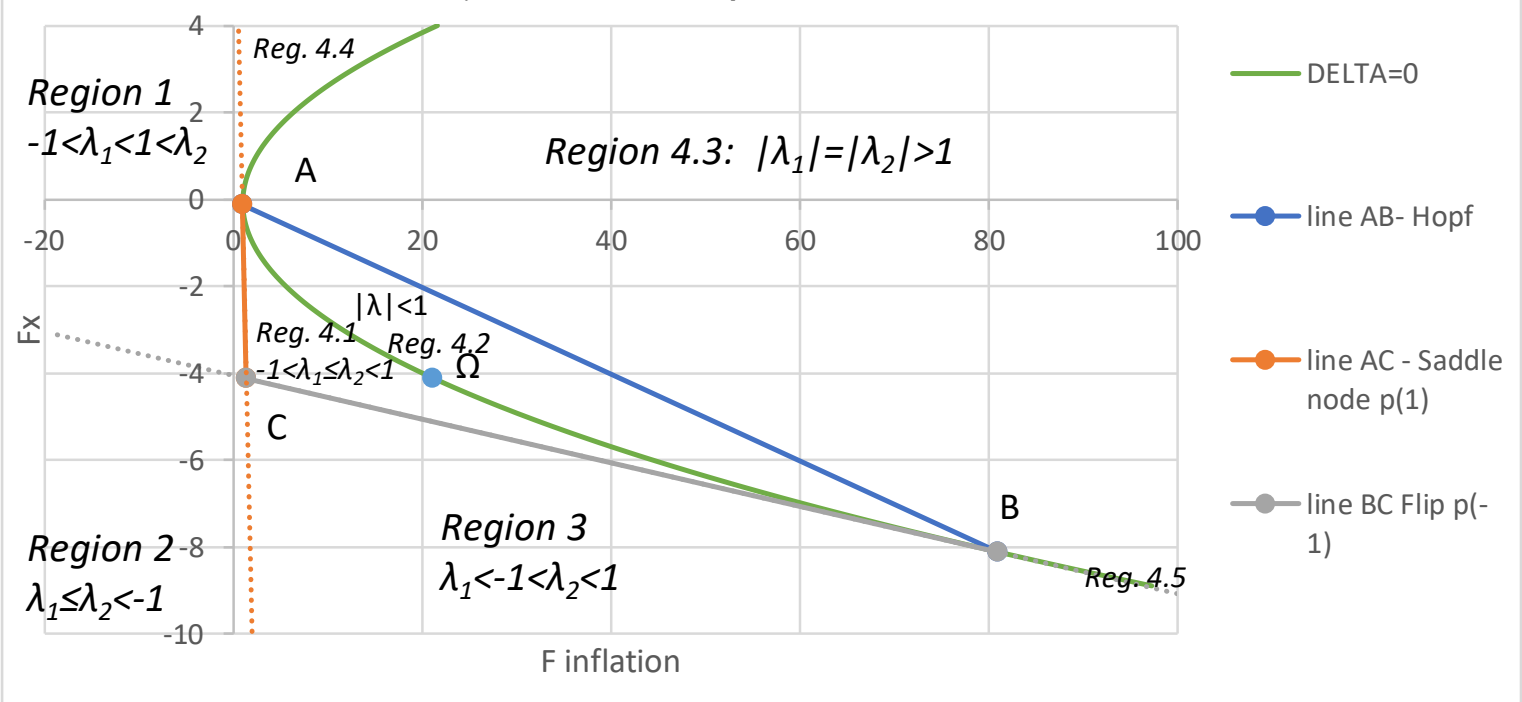

Figure 2: Stability triangle in the plane of policy parameters, $\gamma=-0.5, \kappa=-0.1, \beta=0.99$

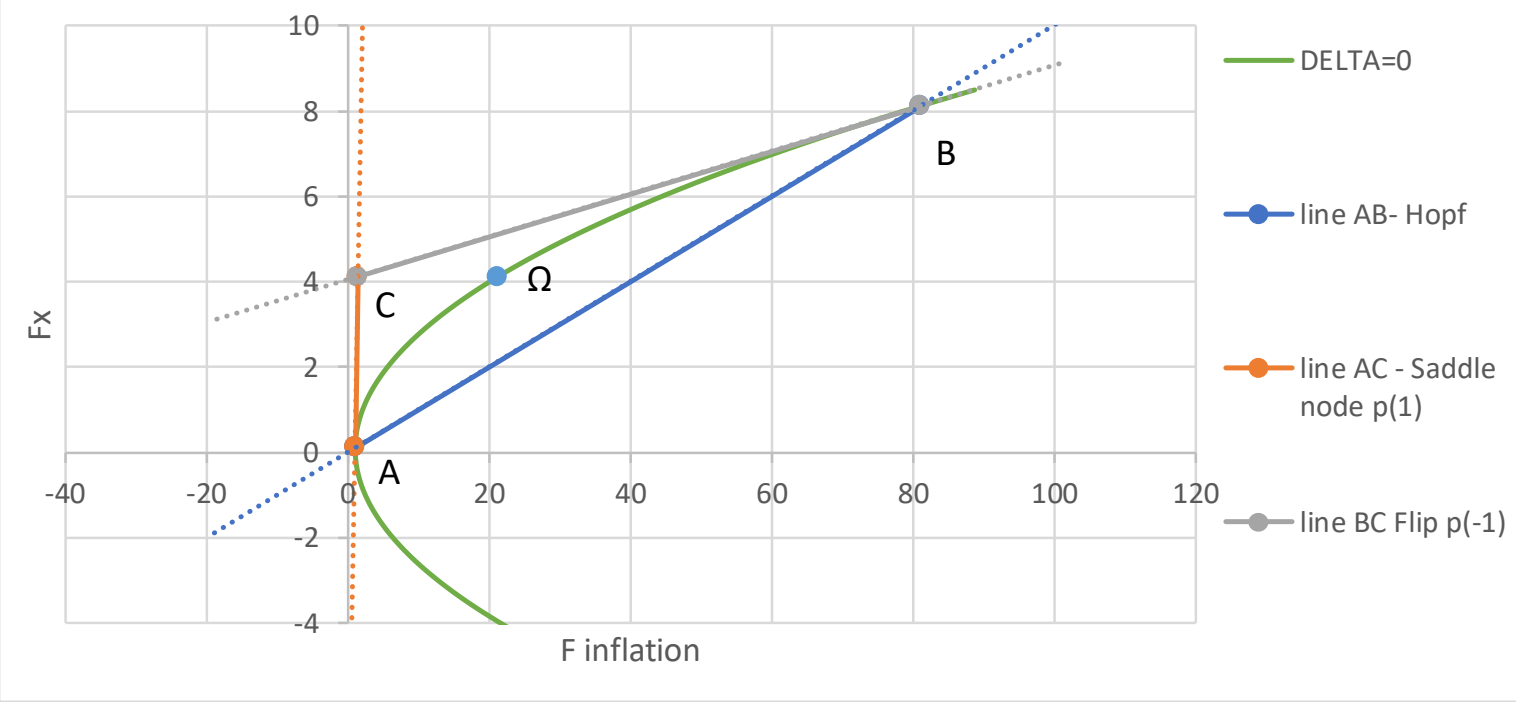


Figure 3: LQR and stability triangle in the plane of policy

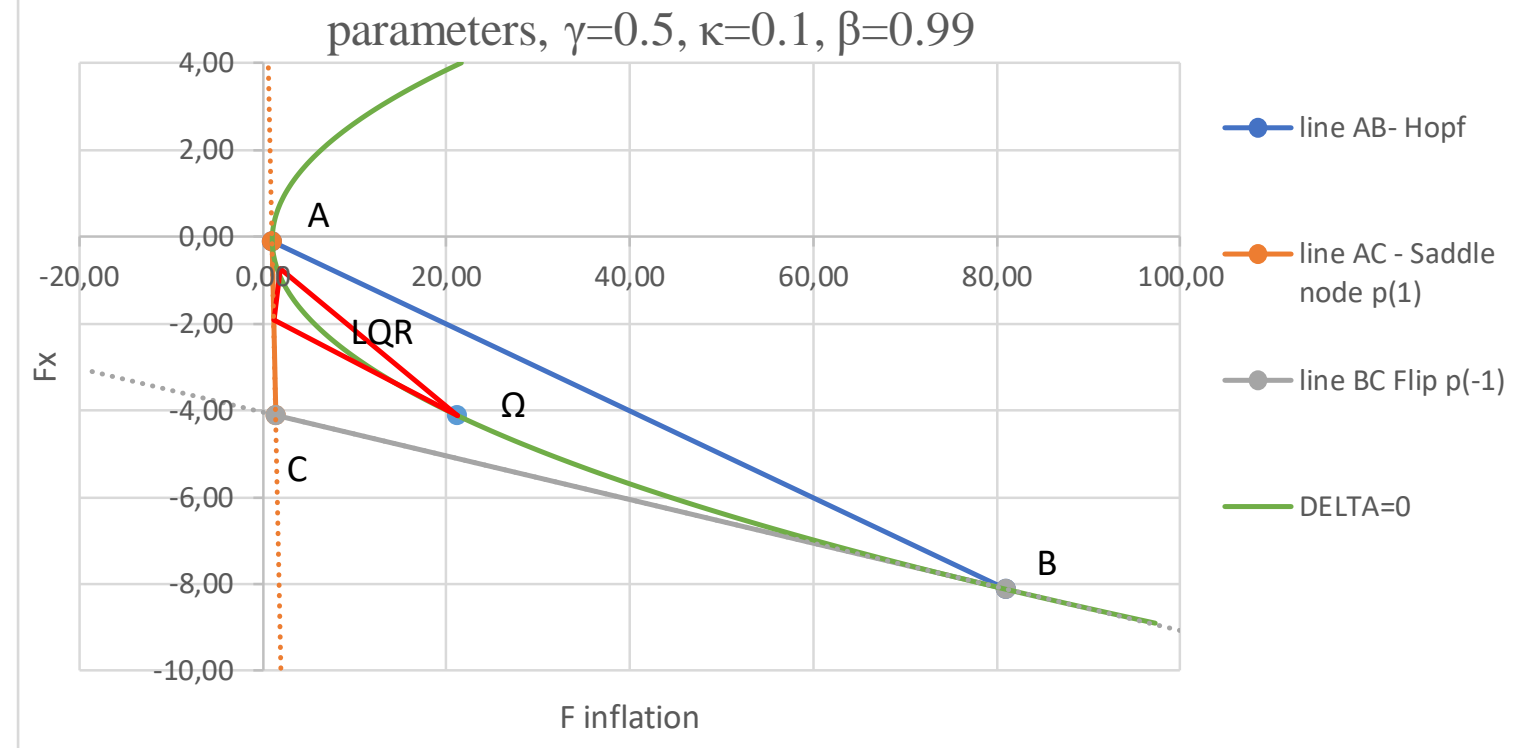

Figure 4: LQR and stability triangle in the plane of policy parameters, $\gamma=-0.5, \kappa=-0.1, \beta=0.99$

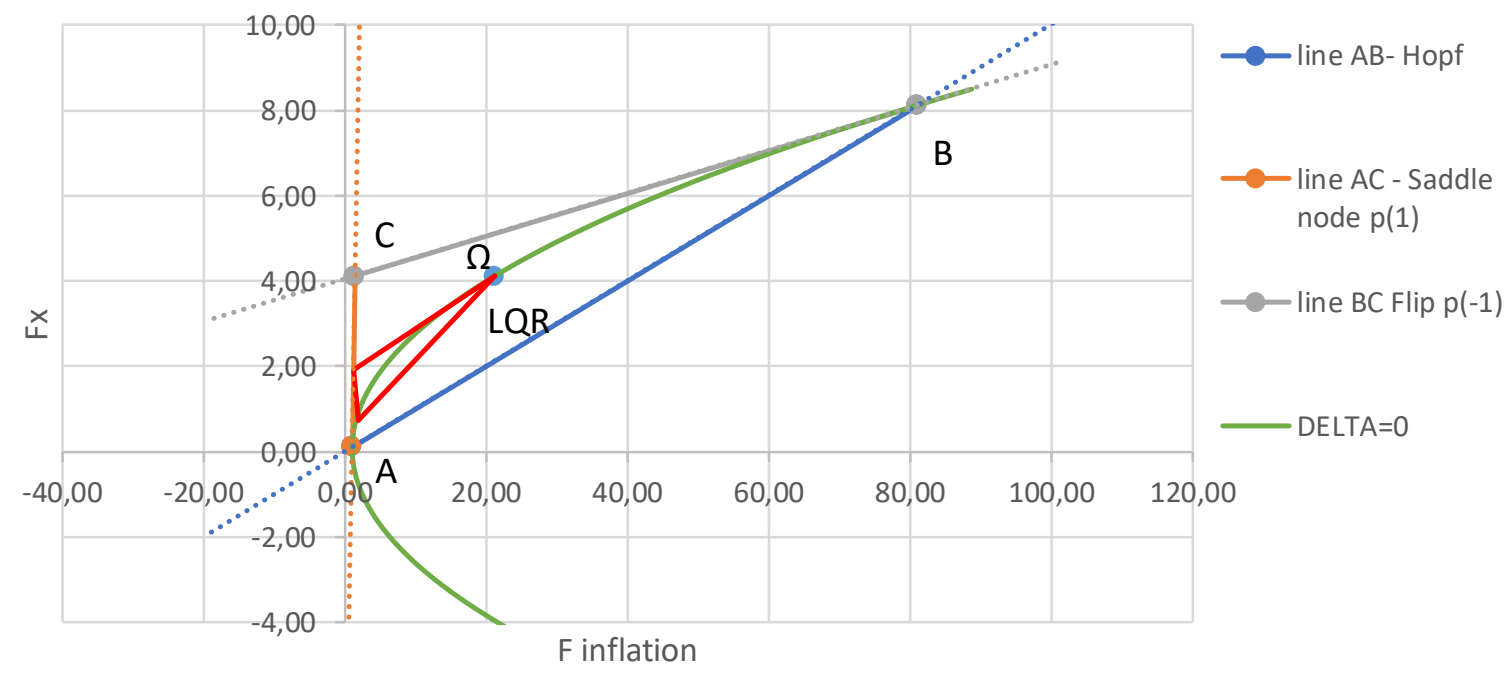


Figure 5: New-Keynesian vs. Ramsey optimal policy, $\gamma=0.5, \kappa=0.1, \beta=0.99$.

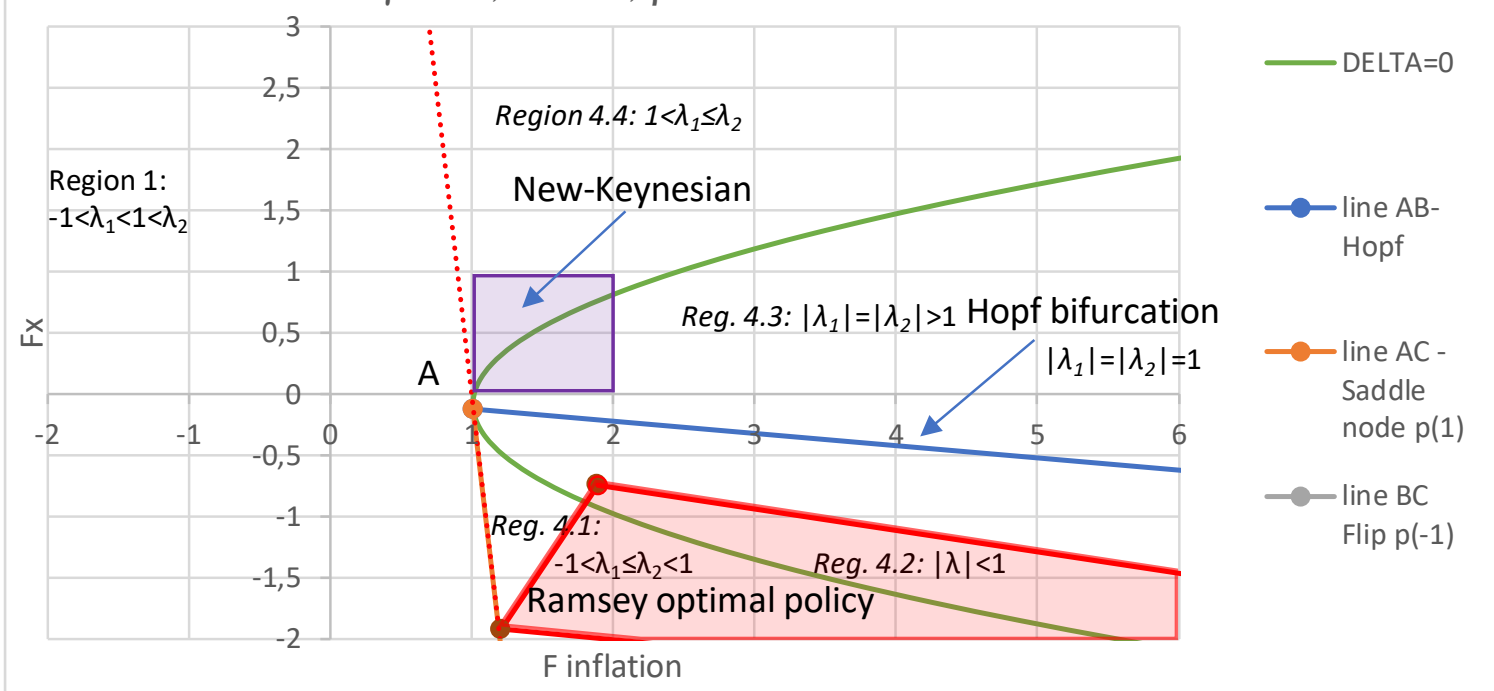

